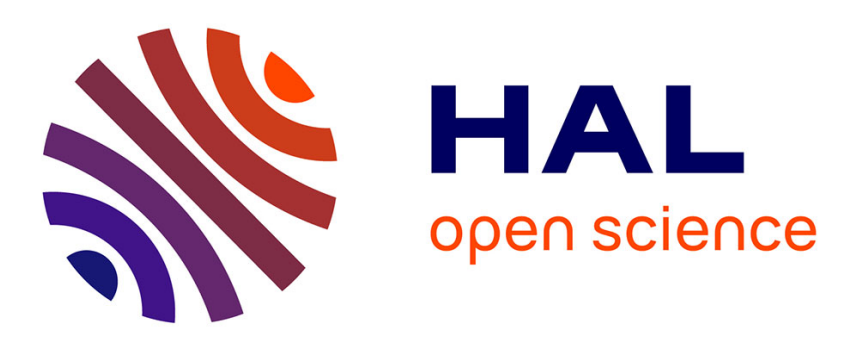

\title{
Modelling and entropy satisfying relaxation scheme for the nonconservative bitemperature Euler system with transverse magnetic field
}

\author{
Stéphane Brull, Xavier Lhébrard, Bruno Dubroca
}

\section{- To cite this version:}

Stéphane Brull, Xavier Lhébrard, Bruno Dubroca. Modelling and entropy satisfying relaxation scheme for the nonconservative bitemperature Euler system with transverse magnetic field. 2019. hal$01939165 \mathrm{v} 2$

\author{
HAL Id: hal-01939165 \\ https://hal.science/hal-01939165v2
}

Preprint submitted on 8 Jan 2019

HAL is a multi-disciplinary open access archive for the deposit and dissemination of scientific research documents, whether they are published or not. The documents may come from teaching and research institutions in France or abroad, or from public or private research centers.
L'archive ouverte pluridisciplinaire HAL, est destinée au dépôt et à la diffusion de documents scientifiques de niveau recherche, publiés ou non, émanant des établissements d'enseignement et de recherche français ou étrangers, des laboratoires publics ou privés. 


\title{
Modelling and entropy satisfying relaxation scheme for the nonconservative bitemperature Euler system with transverse magnetic field
}

\author{
Stéphane Brull * Xavier Lhébrard† Bruno Dubroca $†$
}

November 6, 2018

Revised version submitted to Computers \& Fluids

\begin{abstract}
The present paper concerns the study of the nonconservative bitemperature Euler system with transverse magnetic field. We firstly introduce an underlying conservative kinetic model coupled to Maxwell equations. The nonconservative bitemperature Euler system with transverse magnetic field is then established from this kinetic model by hydrodynamic limit. Next we present the derivation of a finite volume method to approximate weak solutions. It is obtained by solving a relaxation system of Suliciu type, and is similar to HLLC type solvers. The solver is shown in particular to preserve positivity of density and internal energies. Moreover we use a local minimum entropy principle to prove discrete entropy inequalities, ensuring the robustness of the scheme.
\end{abstract}

Keywords: BGK models, hydrodynamic limit, relaxation method, nonconservative hyperbolic system, discrete entropy inequalities, discrete entropy minimum principle

Mathematics Subject Classification: 65M08, 35L60, 65M12

\section{Introduction}

The present paper is devoted to the formal derivation and the approximation of the nonconservative bitemperature Euler system with transverse magnetic field.

This fluid model consists of four conservation equations for mass, momentum (two components), magnetic field, and two nonconservative equations for energies. Physically, this model describes the interaction of a mixture of one species of ions and one species of electrons in thermal nonequilibrium, subjected to a transverse variable magnetic field. The pressure of each species is supposed to satisfy a gamma-law with its own $\gamma$ constant. Moreover the system owns a

\footnotetext{
${ }^{*}$ Université de Bordeaux, Institut de Mathématiques de Bordeaux, UMR 5251, F-33405 Talence, France. (Stephane.Brull@u-bordeaux.fr)

†Université de Bordeaux, Centre de Lasers Intenses et Applications, UMR 5107, F- 33400 Talence, France. (Bruno.Dubroca@u-bordeaux.fr), (Xavier.Lhebrard@u-bordeaux.fr)
} 
dissipative strictly convex entropy, closely related to the classical entropy for the Euler sytem [28].

Solving nonconservative hyperbolic systems is a delicate problem because the definition of weak solutions remains unclear. In order to define nonconservative products, Dal Maso, Lefloch and Murat proposed in [18] a new theory based on the definition of family of paths. In [22], path-conservative schemes are defined by using the concepts developed in [18]. However, it is shown, in [1], that even if the correct path is known, the numerical solution can be far from the expected solution. Let us mention some works dealing with nonconservative Euler type system, where the magnetic fields are neglected. In [5], the authors consider an hyperbolic system having $n-p$ equations in a conservative form, the remaining $p$ equations being nonconservative. In [23], the authors use a Roe solver, and an HLLC solver which neglects the nonconservative part of the system. They validate the approach by comparing their results to theoretical temperatures/pressures curves ( [26], [25]). In [17], the authors assume that the electronic entropy is conserved by all weak solutions including shocks and the system is approached by a system of conservation laws.

Despite these difficulties, the nonconservative formulation is physically relevant. Indeed we show that the bitemperature MHD system admits an underlying conservative kinetic model, which consists of a BGK model coupled with Maxwell equation in the quasi-neutral regime. This result generalizes the approach of [2] in the case of a transverse magnetic field. This BGK model possesses different interspecies collision frequencies in order to take into account discrepancies in the masses of the particles. This point has been in particular mentioned in [19], where a conservative formulation is suggested. Hence following this idea we perform an hydrodynamic limit and we formally get the nonconservative MHD system.

In another way, the present bitemperature MHD system is approached numerically. An important issue in multidimensional simulations is to minimize the numerical viscosity by using accurate solvers, in particular on contact discontinuities; while being robust, see for example [20,30,6].

If dependency is only one spatial variable $x$, the nonconservative two species Euler equations with transverse magnetic field are given by:

$$
\begin{array}{rrrr}
\partial_{t} \rho+\partial_{x} \rho u_{1} & = & 0, \\
\partial_{t}\left(\rho u_{1}\right)+\partial_{x}\left(\rho u_{1}^{2}+p_{e}+p_{i}+B_{3}^{2} / 2\right) & & = \\
\partial_{t}\left(\rho u_{2}\right)+\partial_{x}\left(\rho u_{2} u_{1}\right) & & 0, \\
\partial_{t} \overline{\varepsilon_{e}}+\partial_{x}\left(u_{1}\left(\overline{\varepsilon_{e}}+p_{e}+c_{e} B_{3}^{2} / 2\right)\right) & -u_{1} \partial_{x}\left(c_{i} p_{e}-c_{e} p_{i}\right)= & \widetilde{S}_{e i}, \\
\partial_{t} \overline{\varepsilon_{i}}+\partial_{x}\left(u_{1}\left(\overline{\varepsilon_{i}}+p_{i}+c_{i} B_{3}^{2} / 2\right)\right) & +u_{1} \partial_{x}\left(c_{i} p_{e}-c_{e} p_{i}\right)= & \widetilde{S}_{i e}, \\
\partial_{t} B_{3}+\partial_{x}\left(u_{1} B_{3}\right) & & =0,
\end{array}
$$

where $\rho=\rho_{e}+\rho_{e}$ is the total density of the plasma, $\vec{u}=\left(u_{1}, u_{2}\right)$ is the average velocity of the plasma, $B_{3}$ is the vertical component of the magnetic field. We denote by $T_{e}, T_{i}$ the temperatures of electrons and ions, $\rho_{e}=n_{e} m_{e}, \rho_{i}=n_{i} m_{i}$ the densities of the electrons and ions, $n_{e}, n_{i}$ the concentrations of the electrons and ions, $m_{e}, m_{i}$, the masses of the electrons and ions particles. The source terms for exchanges between electron and ion species $S_{e i}$ and $S_{i e}$ are defined by

$$
\widetilde{S}_{e i}=\widetilde{\nu}_{1, e i}\left(T_{i}-T_{e}\right)+\widetilde{\nu}_{2, e i}\left(\partial_{x} B_{3}\right)^{2}, \quad \widetilde{S}_{i e}=-\widetilde{\nu}_{1, e i}\left(T_{i}-T_{e}\right)+\widetilde{\nu}_{2, i e}\left(\partial_{x} B_{3}\right)^{2},
$$


where $\widetilde{\nu}_{1, e i} \geq 0$ is the frequency exchange between temperatures, $\widetilde{\nu}_{2, e i}, \widetilde{\nu}_{2, i e} \geq 0$ are frequencies due to the drift velocity $u_{2} \pm \partial_{x} B_{3}$. These quantities we will be defined later. The concentrations $n_{e}$ and $n_{i}$ are related by the average ionization number $Z=n_{e} / n_{i} \geq 1$ which is considered here constant. This implies that the mass fractions $c_{\alpha}=\rho_{\alpha} / \rho, \alpha=e, i$ are also constant and $c_{e}$ and $c_{i}$ write

$$
c_{e}=\frac{Z m_{e}}{m_{i}+Z m_{e}}, \quad c_{i}=1-c_{e} .
$$

The electrons and ions pressures and temperatures are related by

$$
p_{e}=n_{e} k_{B} T_{e}, \quad p_{i}=n_{i} k_{B} T_{i}
$$

where $k_{B}$ is the Boltzmann constant. The internal energies are given by

$$
\epsilon_{e}=\frac{k_{B} T_{e}}{m_{e}\left(\gamma_{e}-1\right)}, \quad \epsilon_{i}=\frac{k_{B} T_{i}}{m_{i}\left(\gamma_{i}-1\right)},
$$

where $\gamma_{e}, \gamma_{i}$ are constant numbers belonging to the interval [1,3]. The quantity

$$
\overline{\mathcal{E}_{\alpha}}=\rho_{\alpha} \varepsilon_{\alpha}+\frac{1}{2} \rho_{\alpha}\left(u_{1}^{2}+u_{2}^{2}\right)+c_{\alpha} B_{3}^{2} / 2
$$

is the total energy associated to each species $\alpha=e, i$. This model is closely related to the model derived in [2], the novelty here is to take as an additional variable the vertical component of the magnetic field $B_{3}$.

The homogeneous system associated to (1.1) - (1.6) is endowed with an entropy inequality:

$$
\partial_{t}\left(-\rho\left(s_{e}+s_{i}\right)\right)+\partial_{x}\left(-\rho u\left(s_{e}+s_{i}\right)\right) \leq 0,
$$

where $s_{\alpha}, \alpha=e, i$ is the classical specific entropy

$$
s_{\alpha}\left(\rho, \varepsilon_{\alpha}\right)=\frac{c_{\alpha}}{m_{\alpha}\left(\gamma_{\alpha}-1\right)} \ln \left(\frac{\left(\gamma_{\alpha}-1\right) \varepsilon_{\alpha}}{\rho^{\left(\gamma_{\alpha}-1\right)}}\right)+C .
$$

Here $C$ is a nonnegative constant.

The homogeneous system associated to (1.1) - (1.6) is an hyperbolic system. The three eigenvalues of the system are $u, u-\sqrt{\left(\gamma_{e} p_{e}+\gamma_{i} p_{i}+B_{3}^{2}\right) / \rho}$ and $u+\sqrt{\left(\gamma_{e} p_{e}+\gamma_{i} p_{i}+B_{3}^{2}\right) / \rho}$ of multiplicity four, one and one, respectively. The eigenvalue $u$ is linearly degenerate and the associated contact discontinuities is called a material contact. The jump relation associated is as follows. Across a material contact, the quantities $u_{1}$ and $p_{e}+p_{i}+B_{3}^{2} / 2$ are constant. We note here that unlike the 7 -wave full MHD system [11] or the 5 -wave shallow water MHD system [13], the transverse magnetic system has a 3-wave structure and does not admit Alfven waves.

A finite volume scheme for this homogeneous quasilinear system is classically built following Godunov's approach, by considering piecewise constant approximation of

$$
U=\left(\rho, \rho u_{1}, \rho u_{2}, \mathcal{E}_{e}+c_{e} B_{3}^{2} / 2, \mathcal{E}_{i}+c_{i} B_{3}^{2} / 2, B_{3}\right) \in \mathbb{R}^{6}
$$

and invoking an approximate Riemann solver at the interface between two cells, see for example [21] or [9, Section 2.3]. A difficulty is however that the system 
is not conservative. In this paper we apply the relaxation approach of $[10,9$, $11,12,16]$ to the bitemperature transverse MHD system, in order to get an approximate Riemann solver that is entropy preserving, ensuring robustness, while being exact on isolated material contacts. The relaxation system is of Suliciu type as introduced in [27], and the approximate Riemann solver belongs to the family of HLLC solvers, as in $[21,9,4,24,7,20,6]$.

Let us emphasize that the discrete entropy inequalities are established by arguing a suitable extension of technique introduced in [7], also used in the context of solving the ten moment equations [8]. Put in other words, at the discrepancy with the previous works $[10,9]$, an entropy extension is obtained implicitly and thus the entropy dissipation is not evaluated in the proposed discrete entropy inequalities.

This work is organized as follows. In the next section we present the kinetic model involved in this paper. Firstly we consider the Vlasov-BGK model from which the MHD system is derived. Starting from an ad-hoc scaling the construction of the MHD system is performed. In section 3 we derive a relaxation scheme. In section 4 we establish the stability of our scheme. Numerical tests are performed in section 5 to illustrate both accuracy and robustness of the proposed scheme in 1D.

\section{Kinetic model}

Kinetic models are described by the distribution function $f_{\alpha}$ of each species depending on the time variable $t \in \mathbb{R}_{+}$, on the positions $x \in \mathbb{R}^{3}$ and on the velocity $v \in \mathbb{R}^{3}$. The macroscopic quantities can be obtained by extracting moments on these distribution function w.r.t. the velocity variable. Indeed density, velocity and total energy of the species $\alpha$ can be defined as

$$
\begin{gathered}
n_{\alpha}=\int_{R^{3}} f_{\alpha} d v, \quad u_{\alpha}=\frac{1}{n_{\alpha}} \int_{R^{3}} v f_{\alpha} d v, \\
\mathcal{E}_{\alpha}=\frac{3}{2} \rho_{\alpha} \frac{k_{B}}{m_{\alpha}} T_{\alpha}+\frac{1}{2} \rho_{\alpha} u_{\alpha}^{2}=\int_{R^{3}} m_{\alpha} \frac{|v|^{2}}{2} f_{\alpha} d v,
\end{gathered}
$$

where $m_{\alpha}$ is the mass particle, $\rho_{\alpha}=m_{\alpha} n_{\alpha}$, and $T_{\alpha}$ is the temperature of species $\alpha$. The current in the plasma $j$ and the total charge $\bar{\rho}$ are defined by

$$
\begin{gathered}
\bar{\rho}=\int_{\mathbb{R}^{3}}\left(q_{e} f_{e}+q_{i} f_{i}\right) d v=n_{e} q_{e}+n_{i} q_{i}, \\
j=\int_{\mathbb{R}^{3}} v\left(q_{e} f_{e}+q_{i} f_{i}\right) d v=n_{e} q_{e} u_{e}+n_{i} q_{i} u_{i} .
\end{gathered}
$$

\subsection{Kinetic model for a transverse magnetic field}

In this section, we present the BGK model developed in this paper describing a plasma interacting with an electric field $E=\left(E_{1}, E_{2}, 0\right) \in \mathbb{R}^{3}$ and a magnetic field $B=\left(0,0, B_{3}\right) \in \mathbb{R}^{3}$. The model writes

$$
\begin{aligned}
& \partial_{t} f_{\alpha}+v_{1} \partial_{x} f_{\alpha}+\frac{q_{\alpha}}{m_{\alpha}}(\left.E_{1}+B_{3} v_{2}\right) \frac{\partial f_{\alpha}}{\partial v_{1}}+\frac{q_{\alpha}}{m_{\alpha}}\left(E_{2}-B_{3} v_{1}\right) \frac{\partial f_{\alpha}}{\partial v_{2}} \\
&=\frac{1}{\tau_{\alpha}}\left(\mathcal{M}_{\alpha}\left(f_{\alpha}\right)-f_{\alpha}\right)+\frac{1}{\tau_{\alpha \beta}}\left(\overline{\mathcal{M}_{\alpha}}\left(f_{\alpha}, f_{\beta}\right)-f_{\alpha}\right)
\end{aligned}
$$


with $\tau_{\alpha}>0, \tau_{\alpha \beta}>0$. We denote by $\frac{1}{\tau_{\alpha}}$ the collision frequency for the interaction between $\alpha$ particles and $\frac{1}{\tau_{\alpha \beta}}$ the collision frequency for the ion/electron interaction. The frequencies $\tau_{e i}$ and $\tau_{i e}$ being of order of the mass ratios, [19] suggested to take $\tau_{e i} \neq \tau_{i e}$. The quantity $q_{\alpha}$ is the charge of the species $\alpha$. Moreover we define the velocity and the temperature of the mixture by

$$
u=\frac{\rho_{e} u_{e}+\rho_{i} u_{i}}{\rho_{e}+\rho_{i}}, \quad T=\frac{\frac{1}{2} \sum_{\alpha} \rho_{\alpha}\left(u_{\alpha}^{2}-u^{2}\right)+\frac{3}{2} \sum_{\alpha} n_{\alpha} k_{B} T_{\alpha}}{\frac{3}{2} n k_{B}} .
$$

Let us highlight that one can check that the temperature of the mixture satisfies $T \geq 0$. Here fictitious velocity and temperature $u^{\#}, T^{\#}$ introduced in [2] are defined by

$$
u^{\#}=\frac{\frac{\rho_{e}}{\tau_{e i}} u_{e}+\frac{\rho_{i}}{\tau_{i e}} u_{i}}{\frac{\rho_{e}}{\tau_{e i}}+\frac{\rho_{i}}{\tau_{i e}}}, \quad T^{\#}=\frac{\frac{1}{2} \sum_{\alpha} \frac{\rho_{\alpha}}{\tau_{\alpha \beta}}\left(u_{\alpha}^{2}-\left(u^{\#}\right)^{2}\right)+\frac{3}{2} k_{B} \sum_{\alpha} \frac{n_{\alpha}}{\tau_{\alpha \beta}} T_{\alpha}}{\frac{3}{2} k_{B}\left(\frac{n_{e}}{\tau_{e i}}+\frac{n_{i}}{\tau_{i e}}\right)} .
$$

The two Maxwellian distribution functions $\mathcal{M}_{\alpha}$ and $\overline{\mathcal{M}_{\alpha}}$ are defined by:

$$
\mathcal{M}_{\alpha}\left(f_{\alpha}\right)=\frac{n_{\alpha}}{\left(2 \pi k_{B} T_{\alpha} / m_{\alpha}\right)^{3 / 2}} \exp \left(-\frac{\left(v_{1}-u_{1, \alpha}\right)^{2}+\left(v_{2}-u_{2, \alpha}\right)^{2}+v_{3}^{2}}{2 k_{B} T_{\alpha} / m_{\alpha}}\right),
$$

and

$$
\overline{\mathcal{M}_{\alpha}}\left(f_{\alpha}\right)=\frac{n_{\alpha}}{\left(2 \pi k_{B} T^{\#} / m_{\alpha}\right)^{3 / 2}} \exp \left(-\frac{\left(v_{1}-u_{1}^{\#}\right)^{2}+\left(v_{2}-u_{2}^{\#}\right)^{2}+v_{3}^{2}}{2 k_{B} T^{\#} / m_{\alpha}}\right) .
$$

The two Maxwellian distributions satisfy the constraints

$$
\int_{\mathbb{R}^{3}}\left(\mathcal{M}_{\alpha}-f_{\alpha}\right)\left[\begin{array}{l}
m_{\alpha} \\
m_{\alpha} v \\
m_{\alpha} \frac{v^{2}}{2}
\end{array}\right] d v=0, \quad \int_{\mathbb{R}^{3}}\left(\overline{\mathcal{M}_{\alpha}}-f_{\alpha}\right) d v=0
$$

and

$$
\int_{\mathbb{R}^{3}}\left(\frac{1}{\tau_{e i}}\left(\mathcal{M}_{\alpha}-f_{\alpha}\right)\left[\begin{array}{l}
m_{\alpha} v \\
m_{\alpha} \frac{v^{2}}{2}
\end{array}\right]+\frac{1}{\tau_{i e}}\left(\overline{\mathcal{M}_{\alpha}}-f_{\alpha}\right)\left[\begin{array}{l}
m_{\alpha} v \\
m_{\alpha} \frac{v^{2}}{2}
\end{array}\right]\right) d v=0 .
$$

This model is coupled with the Maxwell equations which write in the transverse magnetic field

$$
\begin{aligned}
\partial_{x} E_{1} & =\frac{\bar{\rho}}{\varepsilon_{0}}, & \partial_{t} E_{1} & =-\frac{j_{1}}{\varepsilon_{0}}, \\
\partial_{t} B_{3}+\partial_{x} E_{2} & =0, & \partial_{t} E_{2}-c^{2} \partial_{x} B_{3} & =-\frac{j_{2}}{\varepsilon_{0}},
\end{aligned}
$$

where $j=\left(j_{1}, j_{2}\right)$ has been defined in $(2.2), c$ represents the speed of light and $\varepsilon_{0}$ is the vacuum permittivity. 


\subsection{Hydrodynamic limit}

\subsubsection{Scaling on the one dimensionnal BGK model}

In order to use a Chapman-Enskog procedure, we introduce a small parameter $\kappa$ and the BGK model (2.3) is rescaled as:

$$
\begin{aligned}
\partial_{t} f_{\alpha}+v_{1} \partial_{x} f_{\alpha}+ & \frac{q_{\alpha}}{m_{\alpha}}\left(E_{1}+B_{3} v_{2}\right) \frac{\partial f_{\alpha}}{\partial v_{1}}+\frac{q_{\alpha}}{m_{\alpha}}\left(E_{2}-B_{3} v_{1}\right) \frac{\partial f_{\alpha}}{\partial v_{2}} \\
& =\frac{1}{\kappa}\left(\mathcal{M}_{\alpha}\left(f_{\alpha}\right)-f_{\alpha}\right)+\frac{1}{\tau_{\alpha \beta}}\left(\overline{\mathcal{M}_{\alpha}}\left(f_{\alpha}, f_{\beta}\right)-f_{\alpha}\right) .
\end{aligned}
$$

Moreover this model is coupled with Maxwell equations (2.10), which are rescaled as:

$$
\begin{aligned}
\partial_{x} E_{1} & =\frac{\bar{\rho}}{\kappa^{2}}, & \partial_{t} E_{1} & =-\frac{j_{1}}{\kappa^{2}}, \\
\partial_{t} B_{3}+\partial_{x} E_{2} & =0, & \partial_{t} E_{2}+\frac{1}{\varepsilon^{2}} \partial_{x} B_{3} & =-\frac{j_{2}}{\kappa^{2}} .
\end{aligned}
$$

\subsubsection{Derivation of Euler equations}

In a previous work [2], the nonconservative bitemperature Euler system has been established as the hydrodynamic limit of a conservative kinetic system. In particular the authors deal with the case $B_{3}=0$, which implies that the current $j=\left(j_{1}, j_{2}\right)$ at equilibrium satisfies $j=0$ and enables to express the electric field as a combination of first order spatial derivatives of the variables $\rho, T_{e}, T_{i}$.

The novelty in our case is that we have $j_{1}=0$ and $j_{2}=-\partial_{x} B_{3} \neq 0$. Thus we have a slightly different approach and we proceed in two steps.

- First we perform an hydrodynamic limit of (2.11) -(2.12). The first component $E_{1}$ will behave as in [2] and will be expressed as a combination of first order spatial derivatives of the variables $\rho, u_{2}, T_{e}, T_{i}, B_{3}$. However, the evolution of $E_{2}$ will be given by the second order PDE (2.21).

- Second we use the smallness of the mass ratio $m_{e} / m_{i}$ which enables to simplify the equation satisfied by $E_{2}$.

After those two steps we recover the bitemperature Euler system with transverse magnetic field (1.1)-(1.6).

Proposition 2.1. The kinetic conservative system (2.11) - (2.7) converges formally to the following system

$$
\begin{aligned}
\partial_{t} \rho & +\partial_{x} \rho u_{1}=0 \\
\partial_{t}\left(\rho u_{1}\right) & +\partial_{x}\left(\rho u_{1}^{2}+p_{e}+p_{i}+B_{3}^{2} / 2\right)=0 \\
\partial_{t}\left(\rho u_{2}\right) & +\partial_{x}\left(\rho u_{2} u_{1}\right)=0 \\
\partial_{t}\left(\varepsilon_{e}+c_{e} B_{3}^{2} / 2\right) & +\partial_{x}\left(u_{1}\left(\mathcal{E}_{e}+p_{e}\right)+c_{e} E_{2} B_{3}\right)-u_{1} \partial_{x}\left(c_{i} p_{e}-c_{e} p_{i}\right) \\
& -\left(E_{2}-u_{1} B_{3}\right)\left(q_{e} n_{e} u_{2}+\left(c_{e}-c_{i}\right) \partial_{x} B_{3}\right)=S_{e i}, \\
\partial_{t}\left(\varepsilon_{i}+c_{i} B_{3}^{2} / 2\right) & +\partial_{x}\left(u_{1}\left(\varepsilon_{i}+p_{i}\right)+c_{i} E_{2} B_{3}\right)+u_{1} \partial_{x}\left(c_{i} p_{e}-c_{e} p_{i}\right) \\
& +\left(E_{2}-u_{1} B_{3}\right)\left(q_{e} n_{e} u_{2}+\left(c_{e}-c_{i}\right) \partial_{x} B_{3}\right)=-S_{e i}, \\
\partial_{t} B_{3} & +\partial_{x} E_{2}=0,
\end{aligned}
$$


where

$$
\varepsilon_{\alpha}=\rho_{\alpha} \varepsilon_{\alpha}+\frac{1}{2} \rho_{\alpha}\left(u_{1}^{2}+u_{2, \alpha}^{2}\right), \quad \alpha=e, i
$$

with $u_{2, \alpha}$ defined by (2.33). Moreover the first component of electric field $E_{1}$ is explicitly given by the Ohm's law

$$
c_{i} \partial_{x} p_{e}-c_{e} \partial_{x} p_{i}+\left(c_{i}-c_{e}\right) \partial_{x}\left(B_{3}^{2} / 2\right)=q_{e} n_{e}\left(E_{1}+u_{2} B_{3}\right),
$$

and the second component of electric field $E_{2}$ satisfies the following equation:

$$
\partial_{t}\left(\partial_{x} B_{3}\right)+\partial_{x}\left(u_{1} \partial_{x} B_{3}\right)+\left(q_{e} n_{e}\right)^{2} \frac{\rho}{\rho_{e} \rho_{i}}\left(E_{2}-B_{3} u_{1}\right)=-\frac{\partial_{x} B_{3}}{\tau_{i e} c_{e}+\tau_{e i} c_{i}} .
$$

In addition the source term $S_{e i}$ is defined by

$$
S_{e i}=\nu_{1, e i}\left(T_{i}-T_{e}\right)+\nu_{2, e i}\left(\partial_{x} B_{3}\right)^{2}+\nu_{3, e i} u_{2} \partial_{x} B_{3}
$$

with the coefficients $\nu_{1, e i}, \nu_{2, e i}, \nu_{3, e i}$ given by

$$
\begin{gathered}
\nu_{1, e i}=\frac{3}{2} \frac{k_{B} n_{e} n_{i}}{\tau_{i e} n_{e}+\tau_{e i} n_{i}} \\
\nu_{2, e i}=c_{e} c_{i} \rho \frac{\left(c_{e}-c_{i}\right)\left(n_{i} c_{i} \tau_{e i}^{2}+n_{e} c_{e} \tau_{i e}^{2}\right)-2 c_{e} c_{i}\left(n_{i}-n_{e}\right) \tau_{e i} \tau_{i e}}{\left(\tau_{i e} n_{e}+\tau_{e i} n_{i}\right)\left(q_{e} n_{e}\right)^{2}\left(\tau_{i e} c_{e}+\tau_{e i} c_{i}\right)^{2}}
\end{gathered}
$$

and

$$
\nu_{3, e i}=\frac{c_{e} c_{i} \rho}{\left(q_{e} n_{e}\right)\left(c_{e} \tau_{i e}+c_{i} \tau_{e i}\right)}
$$

Note that with Proposition 2.1, we do not recover yet (1.1) - (1.6). Using the following approximation we are able to simplify the previous system and recover the bitemperature Euler system with transverse magnetic field.

Proposition 2.2. We consider the previous system (2.13)-(2.25). Using the approximation of a small mass ratio between electron and ion species $m_{e} / m_{i}=$ $\kappa<<1$, the equation (2.21) formally converges to

$$
E_{2}=B_{3} u_{1}
$$

Thus in this setting the conservative kinetic system (2.11) - (2.7) formally converges to the nonconservative bitemperature MHD system (1.1) - (1.6). The electric field $E=\left(E_{1}, E_{2}\right)$ is given by the Ohm's laws (2.20) and (2.26). The exchange coefficient $\widetilde{\nu}_{1, e i}=\nu_{1, e i}$ is defined by (2.23). The magnetic coefficients $\widetilde{\nu}_{2, e i}, \widetilde{\nu}_{2, i e}$ are defined by

$$
\begin{aligned}
& \widetilde{\nu}_{2, e i}=c_{e} c_{i} \rho \frac{\left(\tau_{i e} n_{e}+\tau_{e i} n_{i}\right)\left(\tau_{i e} c_{e}+\tau_{e i} c_{i}\right)+\left(c_{i} n_{e}-c_{e} n_{i}\right) \tau_{e i} \tau_{i e}}{2\left(q_{e} n_{e}\right)^{2}\left(\tau_{i e} n_{e}+\tau_{e i} n_{i}\right)\left(\tau_{i e} c_{e}+\tau_{e i} c_{i}\right)^{2}}, \\
& \widetilde{\nu}_{2, i e}=c_{e} c_{i} \rho \frac{\left(\tau_{i e} n_{e}+\tau_{e i} n_{i}\right)\left(\tau_{i e} c_{e}+\tau_{e i} c_{i}\right)-\left(c_{i} n_{e}-c_{e} n_{i}\right) \tau_{e i} \tau_{i e}}{2\left(q_{e} n_{e}\right)^{2}\left(\tau_{i e} n_{e}+\tau_{e i} n_{i}\right)\left(\tau_{i e} c_{e}+\tau_{e i} c_{i}\right)^{2}} .
\end{aligned}
$$

Remark 2.3. In the case $B_{3}=0$ we recover all the results of [2]. Concerning the Ohms law, compared to [2], in (2.20) we have additional terms $\left(c_{e}-c_{i}\right) \partial_{x}\left(B_{3}^{2} / 2\right)+q_{e} n_{e} u_{2} B_{3}$. 
Proof of Proposition 2.1. For this proof, for any $g$ belonging in $L_{2}^{1}=\{g \in$ $\left.L^{1} /\left(1+v^{2}\right) f \in L^{1}\right\}$, we use the notation

$$
\langle g\rangle=\int_{\mathbb{R}^{3}} g d v
$$

the equilibrium states of (2.11) write

$$
\begin{aligned}
f_{\alpha}=\mathcal{M}_{\alpha}, \quad \alpha=e, i, \quad \bar{\rho}=0, & j_{1}=0 \\
\partial_{t} B_{3}+\partial_{x} E_{2}=0, & j_{2}=-\partial_{x} B_{3} .
\end{aligned}
$$

This system implies that

$$
\begin{aligned}
n_{e} q_{e}+n_{i} q_{i} & =0, \quad n_{e} q_{e} u_{1, e}+n_{i} q_{i} u_{1, i}=0, \\
\partial_{t} B_{3}+\partial_{x} E_{2} & =0, \quad n_{e} q_{e} u_{2, e}+n_{i} q_{i} u_{2, i}=-\partial_{x} B_{3},
\end{aligned}
$$

which is equivalent to

$$
\begin{array}{r}
n_{e} q_{e}+n_{i} q_{i}=0, \\
u_{1, e}=u_{1, i}=u, \\
n_{e} q_{e}\left(u_{2, e}-u_{2, i}\right)=-\partial_{x} B_{3}, \\
\partial_{t} B_{3}+\partial_{x} E_{2}=0,
\end{array}
$$

with $u$ defined by (2.4). Moreover combining (2.4) and (2.31) we compute

$$
\left\{\begin{array}{l}
u_{2, e}=u_{2}-\frac{c_{i}}{q_{e} n_{e}} \partial_{x} B_{3}, \\
u_{2, i}=u_{2}-\frac{c_{e}}{q_{i} n_{i}} \partial_{x} B_{3} .
\end{array}\right.
$$

Next we establish the hydrodynamic limit associated to the previous equilibrium. Hence $f_{\alpha}$ writes

$$
f_{\alpha}=\mathcal{M}_{\alpha}+\kappa g_{\alpha}
$$

with

$$
\int_{\mathbb{R}^{3}} m_{\alpha}\left(\begin{array}{c}
1 \\
|v|^{2}
\end{array}\right) g_{\alpha}=0, \quad \int_{\mathbb{R}^{3}} m_{\alpha}\left(\begin{array}{c}
v_{1} \\
v_{2}
\end{array}\right) g_{\alpha}=0 .
$$

Plugging (2.34) into (2.11), we compute $g_{\alpha}$ as follows

$$
\begin{aligned}
\partial_{t} \mathcal{M}_{\alpha}+v_{1} \partial_{x} \mathcal{M}_{\alpha} & +\frac{q_{\alpha}}{m_{\alpha}}\left(E_{1}+B_{3} v_{2}\right) \partial_{v_{1}} \mathcal{M}_{\alpha}+\frac{q_{\alpha}}{m_{\alpha}}\left(E_{2}-B_{3} v_{1}\right) \partial_{v_{2}} \mathcal{M}_{\alpha} \\
& =-g_{\alpha}+\frac{1}{\tau_{\alpha \beta}}\left(\overline{\mathcal{M}_{\alpha}}-\mathcal{M}_{\alpha}\right)+O(\kappa) .
\end{aligned}
$$

From here we will take the moments of the previous equation. The procedure we use here is very similar to the one detailed in [2]. So we skip some details of computation.

Our goal is to obtain the system (2.13)- (2.18) with $E_{1}, E_{2}$ defined by (2.20), (2.21), respectively.

a) Firstly we proceed similarly to [2]. Multiplying (2.36) by $m_{\alpha}$, integrating w.r.t. $v$ and by summing the two equations for electrons and ions gives (2.13). 
b) Then multiplying (2.36) by $m_{\alpha} v_{1}$ and integrating w.r.t. $v$ leads to

$$
\partial_{t}\left(\rho_{\alpha} u_{1}\right)+\partial_{x}\left(\rho_{\alpha} u_{1}^{2}+p_{\alpha}\right)-q_{\alpha} n_{\alpha}\left(E_{1}+B_{3} u_{2, \alpha}\right)=0 .
$$

By summing for electrons and ions (2.37) and using (2.29), (2.30) we get (2.14). Next considering as in [2] the nonconservative form of (2.37) with the additional term $\frac{q_{\alpha} n_{\alpha}}{\rho_{\alpha}} B_{3} u_{2, \alpha}$, we get the generalized Ohm's law (2.20) for $E_{1}$.

c) Then we multiply by $m_{\alpha} v_{2}$ and we integrate w.r.t. $v$ :

$$
\partial_{t}\left(\rho_{\alpha} u_{2, \alpha}\right)+\partial_{x}\left(\rho_{\alpha} u_{2, \alpha} u_{1}\right)-q_{\alpha} n_{\alpha}\left(E_{2}-B_{3} u_{1}\right)=\frac{\rho_{\alpha}}{\tau_{\alpha \beta}}\left(u_{2}^{\#}-u_{2, \alpha}\right) .
$$

Using (2.29) and the definition of $u^{\#}$ in (2.4), we sum for electrons and ions (2.38) and we get (2.15).

Next we compute a generalized Ohm's law for $E_{2}$. First we use (2.31) and the definition of $u^{\#}$ in (2.5) in order to obtain that

$$
\frac{1}{\tau_{\alpha \beta}}\left(u_{2}^{\#}-u_{2, \alpha}\right)=\frac{c_{\beta}}{c_{\alpha} \tau_{\beta \alpha}+c_{\beta} \tau_{\alpha \beta}}\left(\frac{\partial_{x} B_{3}}{q_{\alpha} n_{\alpha}}\right) .
$$

Then we multiply (2.38) by $q_{\alpha} / m_{\alpha}$ and we use (2.39) to get

$$
\partial_{t}\left(q_{\alpha} n_{\alpha} u_{2, \alpha}\right)+\partial_{x}\left(q_{\alpha} n_{\alpha} u_{2, \alpha} u_{1}\right)-\frac{\left(q_{\alpha} n_{\alpha}\right)^{2}}{\rho_{\alpha}}\left(E_{2}-B_{3} u_{1}\right)=\frac{c_{\beta} \partial_{x} B_{3}}{c_{\alpha} \tau_{\beta \alpha}+c_{\beta} \tau_{\alpha \beta}} .
$$

Finally using (2.29) and $q_{e} n_{e} u_{2, e}+q_{i} n_{i} u_{2, i}=-\partial_{x} B_{3}$, we sum for electrons and ions (2.40) and we obtain (2.21).

d) Then we multiply by $m_{\alpha} \frac{|v|^{2}}{2}$ and we integrate w.r.t. $v$ :

$$
\begin{aligned}
\partial_{t} \mathcal{E}_{\alpha}+\partial_{x}\left(u_{1}\left(\mathcal{E}_{\alpha}+p_{\alpha}\right)\right)-q_{\alpha} n_{\alpha}\left(E_{1}\right. & \left.+B_{3} u_{2, \alpha}\right) u_{1} \\
& -q_{\alpha} n_{\alpha}\left(E_{2}-B_{3} u_{1}\right) u_{2, \alpha}=S,
\end{aligned}
$$

whith $\mathcal{E}_{\alpha}$ defined by (2.19) and

$$
S=\frac{1}{\tau_{\alpha \beta}}\left\langle m_{\alpha} \frac{|v|^{2}}{2}\left(\overline{\mathcal{M}_{\alpha}}-\mathcal{M}_{\alpha}\right)\right\rangle .
$$

Using (2.20) and (2.33) we get

$$
\begin{aligned}
\partial_{t} \mathcal{E}_{\alpha}+\partial_{x}\left(u_{1}\left(\mathcal{E}_{\alpha}+p_{\alpha}\right)\right)+ & u_{1}\left(c_{\alpha} \partial_{x} p_{\beta}-c_{\beta} \partial_{x} p_{\alpha}\right)+u_{1} c_{\alpha} B_{3} \partial_{x} B_{3} \\
& -\left(E_{2}-B_{3} u_{1}\right)\left(q_{\alpha} n_{\alpha} u_{2}-c_{\beta} \partial_{x} B_{3}\right)=S .
\end{aligned}
$$

Moreover, we multiply (2.32) by $c_{\alpha} B_{3}$ and we get

$$
\partial_{t}\left(c_{\alpha} B_{3}^{2} / 2\right)+\partial_{x}\left(c_{\alpha} B_{3} E_{2}\right)-c_{\alpha} E_{2} \partial_{x} B_{3}=0 .
$$

Adding (2.43) and (2.44) we get, for $\alpha \neq \beta$

$$
\begin{aligned}
\partial_{t}\left(\mathcal{E}_{\alpha}+c_{\alpha} B_{3}^{2} / 2\right)+\partial_{x}\left(u_{1}\left(\varepsilon_{\alpha}+p_{\alpha}\right)+c_{\alpha} E_{2} B_{3}\right)+u_{1}\left(c_{\alpha} \partial_{x} p_{\beta}-c_{\beta} \partial_{x} p_{\alpha}\right) \\
-\left(E_{2}-B_{3} u_{1}\right)\left(q_{\alpha} n_{\alpha} u_{2}+\left(c_{\alpha}-c_{\beta}\right) \partial_{x} B_{3}\right)=S .
\end{aligned}
$$


At this point we deal with $S$ defined by (2.42). Using (2.6) and (2.7) we write

$$
S=\frac{3}{2} \frac{k_{B} n_{\alpha}}{\tau_{\alpha \beta}}\left(T^{\#}-T_{\alpha}\right)+\frac{1}{2} \frac{\rho_{\alpha}}{\tau_{\alpha \beta}}\left(\left(u_{2}^{\#}\right)^{2}-u_{2, \alpha}^{2}\right) .
$$

Next by using the definition of $T^{\#}(2.5)$ in (2.46), we get

$$
\begin{aligned}
S & =\frac{3}{2} \frac{k_{B} n_{\alpha} n_{\beta}}{\tau_{\beta \alpha} n_{\alpha}+\tau_{\alpha \beta} n_{\beta}}\left(T_{\beta}-T_{\alpha}\right) \\
& +\frac{1}{2} \frac{n_{\alpha} n_{\beta}}{\tau_{\beta \alpha} n_{\alpha}+\tau_{\alpha \beta} n_{\beta}}\left(m_{\alpha}\left(\left(u_{2}^{\#}\right)^{2}-u_{2, \alpha}^{2}\right)-m_{\beta}\left(\left(u_{2}^{\#}\right)^{2}-u_{2, \beta}^{2}\right)\right) .
\end{aligned}
$$

Now we deal with $\left(u_{2}^{\#}\right)^{2}-u_{2, \alpha}^{2}$. First using (2.33) in the definition of $u^{\#}$ in (2.5) we write

$$
u_{2}^{\#}=u_{2}+\frac{c_{\alpha} c_{\beta} \partial_{x} B_{3}\left(\tau_{\alpha \beta}-\tau_{\beta \alpha}\right)}{\left(q_{\alpha} n_{\alpha}\right)\left(c_{\alpha} \tau_{\beta \alpha}+c_{\beta} \tau_{\alpha \beta}\right)} .
$$

Moreover using (2.33) we can compute $u_{2}^{\#}-u_{2, \alpha}$ and $u_{2}^{\#}+u_{2, \alpha}$ and after some simple computations we get that

$$
\begin{aligned}
m_{\alpha}\left(\left(u_{2}^{\#}\right)^{2}-u_{2, \alpha}^{2}\right)= & \frac{2 m_{\alpha} c_{\beta} \tau_{\alpha \beta}}{q_{\alpha} n_{\alpha}\left(c_{\alpha} \tau_{\beta \alpha}+c_{\beta} \tau_{\alpha \beta}\right)} u_{2} \partial_{x} B_{3} \\
& \quad+\frac{m_{\alpha} c_{\beta}^{2} \tau_{\alpha \beta}\left(\left(c_{\alpha}-c_{\beta}\right) \tau_{\alpha \beta}-2 c_{\alpha} \tau_{\beta \alpha}\right)}{\left(q_{\alpha} n_{\alpha}\right)^{2}\left(c_{\alpha} \tau_{\beta \alpha}+c_{\beta} \tau_{\alpha \beta}\right)^{2}}\left(\partial_{x} B_{3}\right)^{2} .
\end{aligned}
$$

At this point we sum last equality for electron and ion species and we get

$$
\begin{aligned}
\frac{1}{2} \frac{n_{e} n_{i}}{\tau_{i e} n_{e}+\tau_{e i} n_{i}}\left(m_{e}\left(\left(u_{2}^{\#}\right)^{2}-u_{2, e}^{2}\right)\right. & \left.-m_{i}\left(\left(u_{2}^{\#}\right)^{2}-u_{2, i}^{2}\right)\right) \\
& =\nu_{2, e i}\left(u_{2} \partial_{x} B_{3}\right)+\nu_{3, e i}\left(\partial_{x} B_{3}\right)^{2},
\end{aligned}
$$

with $\nu_{2, e i}, \nu_{3, e i}$ defined by $(2.24),(2.25)$.

Plugging (2.48) into (2.47) we get the RHS of (2.16) with $\nu_{1, e i}, \nu_{2, e i}$ and $\nu_{3, e i}$ defined by (2.23), (2.24) and (2.25), which concludes the proof.

Proof of Proposition 2.2. We use Proposition 2.1 and we deal with the system (2.13) - (2.21). In addition we use the small mass ratio assumption $\kappa=m_{e} / m_{i}$. We also use the constant ionization number $Z=n_{e} / n_{i}$ and we get

$$
\frac{\rho}{\rho_{e} \rho_{i}}=\frac{n_{e} m_{e}+n_{i} m_{i}}{n_{e} m_{e} n_{i} m_{i}}=\frac{(\kappa Z+1)}{\left(m_{i} n_{i}\right) \kappa Z}=\mathcal{O}\left(\frac{1}{\kappa}\right) .
$$

Retaining terms of order 0 in (2.21), it leads to (2.26). Moreover using (2.26) in $(2.16)$ - (2.18), we get

$$
\begin{aligned}
\partial_{t}\left(\mathcal{E}_{e}+c_{e} B_{3}^{2} / 2\right) & +\partial_{x}\left(u_{1}\left(\mathcal{E}_{e}+c_{e} B_{3}^{2} / 2+p_{e}+c_{e} B_{3}^{2} / 2\right)\right) \\
& -u_{1} \partial_{x}\left(c_{i} p_{e}-c_{e} p_{i}\right)=S_{e i} \\
\partial_{t}\left(\varepsilon_{i}+c_{i} B_{3}^{2} / 2\right) & +\partial_{x}\left(u_{1}\left(\varepsilon_{i}+c_{i} B_{3}^{2} / 2+p_{i}+c_{i} B_{3}^{2} / 2\right)\right) \\
& +u_{1} \partial_{x}\left(c_{i} p_{e}-c_{e} p_{i}\right)=-S_{e i} \\
\partial_{t} B_{3} & +\partial_{x}\left(u_{1} B_{3}\right)=0
\end{aligned}
$$


with $S_{e i}$ defined by (2.22)-(2.25). To complete the proof we have to recover (1.4)- (1.5) with $\widetilde{\nu}_{1, e i}=\nu_{1, e i}, \nu_{1, e i}$ defined by (2.23), and $\widetilde{\nu}_{2, e i}, \widetilde{\nu}_{2, i e}$ defined by $(2.27),(2.28)$. In the following we focus on the electron energy equation (1.4), the ion energy equation (1.5) is obtained in a similar way. First we notice the following equality:

$$
\overline{\varepsilon_{e}}=\mathcal{E}_{e}+c_{e} B_{3}^{2} / 2+\rho_{e} \frac{u_{2}^{2}}{2}-\rho_{e} \frac{u_{2, e}^{2}}{2} .
$$

From (1.4) we have a PDE on $\varepsilon_{e}+c_{e} B_{3}^{2} / 2$, thus in order to use the previous relation we need PDEs on $\rho_{e} \frac{u_{2}^{2}}{2}$ and $\rho_{e} \frac{u_{2, e}^{2}}{2}$. Thus on the one hand, we use (2.26) and (2.38) in order to get

$$
\partial_{t}\left(\rho_{e} u_{2, e}\right)+\partial_{x}\left(\rho_{e} u_{2, e} u_{1}\right)=\nu_{3, e i} \partial_{x} B_{3},
$$

with $\nu_{3, e i}$ defined by (2.25). Next we multiply by $u_{2, e}$ (2.54) and we use (2.13) in order to get that

$$
\partial_{t}\left(\rho_{e} \frac{u_{2, e}^{2}}{2}\right)+\partial_{x}\left(\rho_{e} \frac{u_{2, e}^{2}}{2} u_{1}\right)=-\frac{c_{i}}{q_{e} n_{e}} \nu_{3, e i}\left(\partial_{x} B_{3}\right)^{2}+\nu_{3, e i} u_{2} \partial_{x} B_{3}
$$

On the other hand, we combine (2.13), (2.15) and we obtain

$$
\partial_{t}\left(\rho_{e} \frac{u_{2}^{2}}{2}\right)+\partial_{x}\left(\rho_{e} \frac{u_{2}^{2}}{2} u_{1}\right)=0 .
$$

Next following (2.53), we add (2.50), (2.56) and we substract (2.55), it leads to (1.4). Moreover we obtain the following formulas for $\widetilde{\nu}_{1, e i}, \widetilde{\nu}_{2, e i}, \widetilde{\nu}_{2, i e}$ :

$$
\widetilde{\nu}_{1, e i}=\nu_{1, e i}, \quad \widetilde{\nu}_{2, e i}=\nu_{2, e i}+\frac{c_{i}}{q_{e} n_{e}} \nu_{3, e i}, \quad \widetilde{\nu}_{2, i e}=-\nu_{2, e i}+\frac{c_{e}}{q_{e} n_{e}} \nu_{3, e i} .
$$

Finally using (2.24), (2.25) one recovers (2.27).

\section{Numerical approximation}

The numerical approximation we use has two steps:

- First step. We use an approximate Riemann solver for the homogeneous system. Let $\bar{u}^{n+1 / 2}$ be the obtained solution.

- Second step. We take the temperatures interaction into account implicitly: the approximate solution of system at time $t^{n+1}$ is defined by

$$
\rho^{n+1}=\bar{\rho}^{n+1 / 2}, u_{1}^{n+1}={\overline{u_{1}}}^{n+1 / 2}, u_{2}^{n+1}={\overline{u_{2}}}^{n+1 / 2}, B_{3}^{n+1}=\bar{B}_{3}^{n+1 / 2}
$$

and

$$
\left\{\begin{array}{l}
\varepsilon_{e}^{n+1}={\overline{\varepsilon_{e}}}^{n+1 / 2}+\Delta t S_{e i}^{n+1}, \\
\varepsilon_{i}^{n+1}={\overline{\varepsilon_{i}}}^{n+1 / 2}-\Delta t S_{e i}^{n+1},
\end{array}\right.
$$

where

$$
S_{e i}^{n+1}=\widetilde{\nu}_{1, e i}\left(T_{i}^{n+1}-T_{e}^{n+1}\right)+\widetilde{\nu}_{2, e i}\left(\left(\partial_{x} B_{3}\right)^{n+1}\right)^{2} .
$$

This system is linear and owns an explicit solution. 
From here we will focus on the first step of the numerical approximation. We will explain how to derive an efficient approximate Riemann solvers for the homogeneous part of the system (1.1) - (1.6).

In order to get those approximate Riemann solvers, we use a standard relaxation approach, introduced in [9] for the gas dynamic equations. This approach has been developed in [11] for the MHD equations, in [3] for shallow elastic fluids, in [13] for shallow water MHD equations, in [2] for the bitemperature Euler system. An abstract general description can be found in [16], and related works are $[14,15]$. This technique enables to naturally handle the entropy inequality (1.8), and also preserves the positivity of density and internal energies.

\subsection{Relaxation approach}

\subsubsection{Approximate Riemann solver}

We introduce new variables $\pi_{e}, \pi_{i}$, the relaxed pressures, and $a$ intended to parametrize the speed. The form of the relaxation system is as follows,

$$
\begin{array}{rlr}
\partial_{t} \rho+\partial_{x} \rho u_{1} & =0, \\
\partial_{t}\left(\rho u_{1}\right)+\partial_{x}\left(\rho u_{1}^{2}+\pi_{e}+\pi_{i}+B_{3}^{2} / 2\right) & =0, \\
\partial_{t}\left(\rho u_{2}\right)+\partial_{x}\left(\rho u_{2} u_{1}\right) & =0, \\
\partial_{t} \overline{\varepsilon_{e}}+\partial_{x}\left(u_{1}\left(\overline{\varepsilon_{e}}+\pi_{e}+c_{e} B_{3}^{2} / 2\right)\right)-u_{1} \partial_{x}\left(c_{i} \pi_{e}-c_{e} \pi_{i}\right) & =0, \\
\partial_{t} \overline{\varepsilon_{i}}+\partial_{x}\left(u_{1}\left(\overline{\varepsilon_{i}}+\pi_{i}+c_{i} B_{3}^{2} / 2\right)\right)+u_{1} \partial_{x}\left(c_{i} \pi_{e}-c_{e} \pi_{i}\right) & =0, \\
\partial_{t} B_{3}+\partial_{x}\left(u_{1} B_{3}\right) & =0, \\
\partial_{t}\left(\rho \pi_{e}\right)+\partial_{x}\left(\rho u_{1} \pi_{e}\right)+c_{e}\left(a^{2}-\rho B_{3}^{2}\right) \partial_{x} u_{1} & =0, \\
\partial_{t}\left(\rho \pi_{i}\right)+\partial_{x}\left(\rho u_{1} \pi_{i}\right)+c_{i}\left(a^{2}-\rho B_{3}^{2}\right) \partial_{x} u_{1} & =0, \\
\partial_{t}(\rho a)+\partial_{x}\left(\rho a u_{1}\right) & =0 .
\end{array}
$$

The approximate Riemann solver can be defined as follows, starting from left and right values $U_{l}, U_{r}$ at an interface.

- Solve the Riemann problem of the system (3.1)-(3.9) with initial data obtained by completing $U_{l}, U_{r}$ by the equilibrium relations

$$
\begin{aligned}
& \pi_{e, L}=p_{e, L} \equiv\left(\gamma_{e}-1\right) \rho_{L} \varepsilon_{e, L}, \\
& \pi_{i, L}=p_{i, L} \equiv\left(\gamma_{i}-1\right) \rho_{L} \varepsilon_{i, L}, \\
& \pi_{e, R}=p_{e, R} \equiv\left(\gamma_{e}-1\right) \rho_{R} \varepsilon_{e, R}, \\
& \pi_{i, R}=p_{i, R} \equiv\left(\gamma_{i}-1\right) \rho_{R} \varepsilon_{i, R},
\end{aligned}
$$

and with suitable positive values of $a_{l}, a_{r}$ that will be discussed further on, essentially in Section 3.2.1.

- Retain in the solution only the variables $\rho, \rho u_{1}, \rho u_{2}, \overline{\varepsilon_{e}}, \overline{\varepsilon_{i}}, B_{3}$. The result is a vector called $R\left(x / t, U_{l}, U_{r}\right)$.

Intuitively, the solver is consistent because of the equations (3.1)-(3.6), that are consistent with the equations (1.1)-(1.6). The specific values used for $a$ do not play any role in this consistency.

The accuracy of the solver on isolated contacts is described by the following lemma. 
Lemma 3.1. The approximate Riemann solver $R\left(x / t, U_{l}, U_{r}\right)$ solves exactly the material contact discontinuities.

Proof. Material contacts are solutions to the bitemperature MHD system (1.1) - (1.6) with $u_{1}, p_{e}+p_{i}+B_{3}^{2} / 2$ constant. These solutions are obviously solutions to the relaxation system (3.1) - (3.8) with $\pi_{e}=p_{e}, \pi_{i}=p_{i}$. Thus for these data, $R$ coincides with the exact solver, which concludes the proof.

\subsubsection{Godunov scheme}

Following the Godunov approach, the numerical scheme can be defined by the approximate Riemann solver as follows. We consider a mesh of cells $\left(x_{i-1 / 2}, x_{i+1 / 2}\right)$, $i \in \mathbb{Z}$, of length $\Delta x_{i}=x_{i+1 / 2}-x_{i-1 / 2}$, discrete times $t_{n}$ with $t_{n+1}-t_{n}=\Delta t$, and cell values $U_{i}^{n}$ approximating the average of $U$ over the cell $i$ at time $t_{n}$. We can then define an approximate solution $U_{a p p r}(t, x)$ for $t_{n} \leq t<t_{n+1}$ and $x \in \mathbb{R}$ by

$$
U_{\text {appr }}(t, x)=R\left(\frac{x-x_{i+1 / 2}}{t-t_{n}}, U_{i}^{n}, U_{i+1}^{n}\right) \text { for } x_{i}<x<x_{i+1},
$$

where $x_{i}=\left(x_{i-1 / 2}+x_{i+1 / 2}\right) / 2$. This definition is coherent under a half CFL condition, formulated as

$$
\begin{aligned}
& x / t<-\frac{\Delta x_{i}}{2 \Delta t} \Rightarrow R\left(x / t, U_{i}, U_{i+1}\right)=U_{i}, \\
& x / t>\frac{\Delta x_{i+1}}{2 \Delta t} \Rightarrow R\left(x / t, U_{i}, U_{i+1}\right)=U_{i+1} .
\end{aligned}
$$

The new values at time $t_{n+1}$ are defined by

$$
U_{i}^{n+1}=\frac{1}{\Delta x_{i}} \int_{x_{i-1 / 2}}^{x_{i+1 / 2}} U_{a p p r}\left(t_{n+1}-0, x\right) d x .
$$

Notice that it is only in this averaging procedure that the choice of the particular pseudo-conservative variable $U$ as (1.10) is involved. We can follow the computations of [9, Section 2.3], the only difference being that the system is not conservative. We obtain the update formula

$$
U_{i}^{n+1}=U_{i}^{n}-\frac{\Delta t}{\Delta x_{i}}\left(\mathcal{F}_{l}\left(U_{i}^{n}, U_{i+1}^{n}\right)-\mathcal{F}_{r}\left(U_{i-1}^{n}, U_{i}^{n}\right)\right)
$$

where

$$
\begin{aligned}
& \mathcal{F}_{l}\left(U_{l}, U_{r}\right)=F\left(U_{l}\right)-\int_{-\infty}^{0}\left(R\left(\xi, U_{l}, U_{r}\right)-U_{l}\right) d \xi, \\
& \mathcal{F}_{r}\left(U_{l}, U_{r}\right)=F\left(U_{r}\right)+\int_{0}^{\infty}\left(R\left(\xi, U_{l}, U_{r}\right)-U_{r}\right) d \xi .
\end{aligned}
$$

The variable $\xi$ stands for $x / t$, and the pseudo-conservative flux is chosen as

$$
\begin{aligned}
& F(U) \equiv\left(\rho u_{1}, \quad \rho u_{1}^{2}+p_{e}+p_{i}+B_{3}^{2} / 2, \quad \rho u_{1} u_{2},\right. \\
& \left.u_{1}\left(\overline{\mathcal{E}_{e}}+p_{e}+c_{e} B_{3}^{2} / 2\right), \quad u_{1}\left(\overline{\mathcal{E}_{i}}+p_{i}+c_{i} B_{3}^{2} / 2\right), \quad u_{1} B_{3}\right) .
\end{aligned}
$$

In (3.15), the fourth and fifth components could be chosen differently since the two energy equations in our system are not conservative. We can remark that the choice of $F$ has no influence on the update formula (3.13). 


\subsubsection{Subcharacteristic condition}

We are interested on necessary stability conditions for smooth solutions. In order to address such an issue, we consider (3.7), (3.8) in which we add righthand-sides:

$$
\begin{aligned}
& \partial_{t}\left(\rho \pi_{e}\right)+\partial_{x}\left(\rho u_{1} \pi_{e}\right)+c_{e}\left(a^{2}-\rho B_{3}^{2}\right) \partial_{x} u_{1}=\frac{\rho}{\kappa}\left(p_{e}-\pi_{e}\right), \\
& \partial_{t}\left(\rho \pi_{i}\right)+\partial_{x}\left(\rho u_{1} \pi_{i}\right)+c_{i}\left(a^{2}-\rho B_{3}^{2}\right) \partial_{x} u_{1}=\frac{\rho}{\kappa}\left(p_{i}-\pi_{i}\right) .
\end{aligned}
$$

On the other hand one can check with straightforward computations that the smooth solutions to (1.1) - (1.6) verify

$$
\begin{gathered}
\partial_{t}\left(\rho p_{e}\right)+\partial_{x}\left(\rho p_{e} u_{1}\right)+\gamma_{e} \rho p_{e} \partial_{x} u_{1}=0 \\
\partial_{t}\left(\rho p_{i}\right)+\partial_{x}\left(\rho p_{i} u_{1}\right)+\gamma_{i} \rho p_{i} \partial_{x} u_{1}=0 .
\end{gathered}
$$

Next when the relaxation parameter $\kappa$ tends to zero one has

$$
\pi_{\alpha}=p_{\alpha}+\delta \kappa+\mathcal{O}\left(\kappa^{2}\right), \quad \alpha=e, i .
$$

Pluggin (3.20) into (3.16) or (3.17) then substracting (3.18)-(3.19) we get that :

$$
\delta=c_{\alpha}\left(\rho B_{3}^{2}+\gamma_{\alpha} \rho p_{\alpha}-a^{2}\right) \partial_{x} u_{1}+\mathcal{O}(\kappa) .
$$

Hence the stability condition needed by the parameter $a$ is

$$
a^{2}>\rho B_{3}^{2}+\gamma_{e} \rho p_{e}+\gamma_{i} \rho p_{i} .
$$

\subsubsection{Intermediate states}

By using the variable $V=\left(\rho, u_{1}, u_{2}, B_{3}, \varepsilon_{e}, \varepsilon_{i}, \pi_{e}, \pi_{i}, a\right)$, one can easily compute the eigenvalues of the system (3.1) - (3.9). They read as $\{u-a / \rho, u, u+a / \rho\}$, where $u$ is an eigenvalue of order 7 . All the fields are linearly degenerated. As a consequence, Rankine-Hugoniot conditions are well-defined (the weak Riemann invariants do not jump through the associated discontinuity), and are equivalent to any conservative formulation.

In the solution to the Riemann problem, the speeds corresponding to the previous eigenvalues will be denoted by

$$
\Sigma_{1}<\Sigma_{2}<\Sigma_{3}
$$

Thus we get a 3 -wave solver with two intermediate states. The variables take the values " $\mathrm{L}$ " for $x / t<\Sigma_{1}$, "L*" for $\Sigma_{1}<x / t<\Sigma_{2}$, "R*" for $\Sigma_{2}<x / t<\Sigma_{3}$, "R" for $\Sigma_{3}<x / t$, see Figure 1. There are 7 strong Riemann invariants for the central wave (i.e. quantities that lie in the kernel of $\partial_{t}+u_{1} \partial_{x}$ ), which are

$$
u_{2}, \quad a, \quad \frac{B_{3}}{\rho}, \quad w_{1, e}, \quad w_{1, i}, \quad w_{2, e}, \quad w_{2, i}
$$

with $w_{1, e}, w_{1, i}, w_{2, e}, w_{2, i}$ defined by

$$
w_{1, \alpha}=\pi_{\alpha}+c_{\alpha} B_{3}^{2} / 2+\frac{a_{1}^{2} c_{\alpha}}{\rho}, \quad w_{2, \alpha}=\varepsilon_{\alpha}+\frac{B_{3}^{2}}{2 \rho}-\frac{\left(\pi_{\alpha}+c_{\alpha} B_{3}^{2} / 2\right)^{2}}{2\left(c_{\alpha} a_{1}\right)^{2}} .
$$


$[! h t b p]$

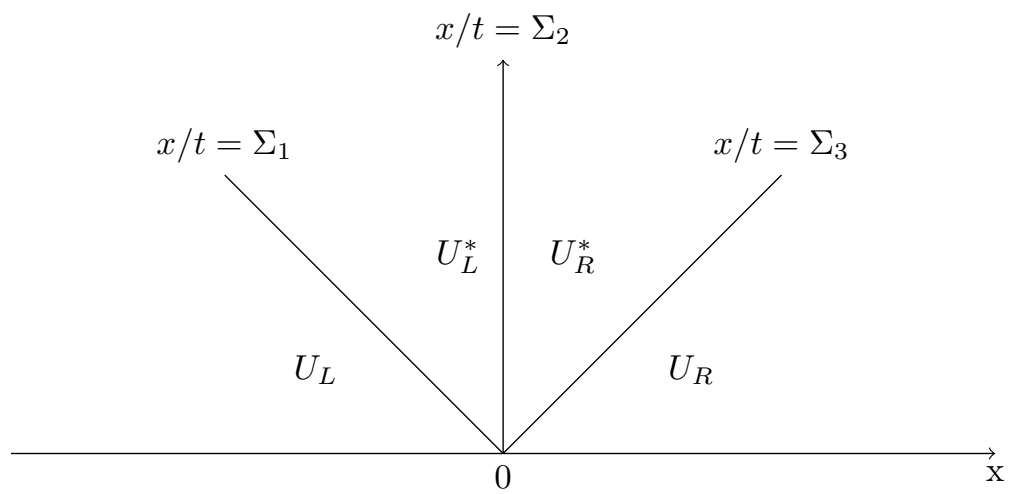

Figure 1: Intermediate states in the Riemann solution

These quantities are thus weak Riemann invariants for the other waves. Two weak Riemann invariants for the central wave are

$$
u_{1}, \quad \pi
$$

with $\pi=\pi_{e}+\pi_{i}+B_{3}^{2} / 2$. We shall denote $u_{1}^{*}$ the common value of $u_{1}$ on the left and on the right of the central wave.

Computations using the Riemann invariants (3.23), (3.24) give the following intermediate states:

$$
\begin{aligned}
& \left\{\begin{array}{l}
\frac{1}{\rho_{L}^{*}}=\frac{1}{\rho_{L}}+\frac{a_{R}\left(u_{1, R}-u_{1, L}\right)+\pi_{L}-\pi_{R}}{a_{L}\left(a_{L}+a_{R}\right)}, \\
\frac{1}{\rho_{R}^{*}}=\frac{1}{\rho_{R}}+\frac{a_{L}\left(u_{1, R}-u_{1, L}\right)+\pi_{R}-\pi_{L}}{a_{R}\left(a_{L}+a_{R}\right)},
\end{array}\right. \\
& u_{1}^{*}=\frac{a_{R} u_{1, R}+a_{L} u_{1, L}+\pi_{L}-\pi_{R}}{a_{L}+a_{R}}, \\
& B_{3, L}^{*}=\frac{\rho_{L}^{*}}{\rho_{L}} B_{3, L}, \quad B_{3, R}^{*}=\frac{\rho_{R}^{*}}{\rho_{R}} B_{3, R},
\end{aligned}
$$

for $\alpha=e, i$ we have

$$
\left\{\begin{array}{l}
\pi_{\alpha, L}^{*}=\pi_{\alpha, L}+\frac{c_{\alpha} B_{3, L}^{2}}{2}\left(1-\left(\frac{\rho_{L}^{*}}{\rho_{L}}\right)^{2}\right)+a_{L}^{2} c_{\alpha}\left(\frac{1}{\rho_{L}}-\frac{1}{\rho_{L}^{*}}\right), \\
\pi_{\alpha, R}^{*}=\pi_{\alpha, R}+\frac{c_{\alpha} B_{3, R}^{2}}{2}\left(1-\left(\frac{\rho_{R}^{*}}{\rho_{R}}\right)^{2}\right)+a_{R}^{2} c_{\alpha}\left(\frac{1}{\rho_{R}}-\frac{1}{\rho_{R}^{*}}\right),
\end{array}\right.
$$




$$
\left\{\begin{aligned}
\varepsilon_{\alpha, L}^{*}=\varepsilon_{\alpha, L}+\frac{B_{3, L}^{2}}{2 \rho_{L}}\left(1-\frac{\rho_{L}^{*}}{\rho_{L}}\right)+ & \frac{\left(\pi_{\alpha, L}^{*}+c_{\alpha} B_{3, L}^{*} / 2\right)^{2}}{2\left(c_{\alpha} a_{L}\right)^{2}}, \\
& -\frac{\left(\pi_{\alpha, L}+c_{\alpha} B_{3, L}^{2} / 2\right)^{2}}{2\left(c_{\alpha} a_{L}\right)^{2}}, \\
\varepsilon_{\alpha, R}^{*}=\varepsilon_{\alpha, R}+\frac{B_{3, R}^{2}}{2 \rho_{R}}\left(1-\frac{\rho_{R}^{*}}{\rho_{R}}\right) & +\frac{\left(\pi_{\alpha, R}^{*}+c_{\alpha} B_{3, R}^{*} / 2\right)^{2}}{2\left(c_{\alpha} a_{R}\right)^{2}} \\
& -\frac{\left(\pi_{\alpha, R}+c_{\alpha} B_{3, R}^{2} / 2\right)^{2}}{2\left(c_{\alpha} a_{R}\right)^{2}} .
\end{aligned}\right.
$$

Finally, using previous formulas one can compute the speeds

$$
\Sigma_{1}=u_{1, L}-\frac{a_{L}}{\rho_{L}}, \quad \Sigma_{2}=u_{1}^{*}, \quad \Sigma_{3}=u_{1, R}+\frac{a_{R}}{\rho_{R}} .
$$

Remark 3.2. Let us notice that $c_{i} \pi_{e}-c_{e} \pi_{i}$ is equal to $c_{i} w_{1, e}-c_{e} w_{1, i}$. As a consequence, $c_{i} \pi_{e}-c_{e} \pi_{i}$ is a Riemann invariant for both extreme eigenvalues. This means that this quantity remains constant through the related contact discontinuities, so that $u \partial_{x}\left(c_{i} \pi_{e}-c_{e} \pi_{i}\right)=0$ there. For the central discontinuity, $u$ is constant so that $u \partial_{x}\left(c_{i} \pi_{e}-c_{e} \pi_{i}\right)=\partial_{x}\left(u\left(c_{i} \pi_{e}-c_{e} \pi_{i}\right)\right)$ this product is also well defined in the usual weak sense.

\section{$3.2 \quad$ Numerical fluxes}

All components of the system except $\overline{\varepsilon_{e}}$ and $\overline{\varepsilon_{i}}$ are conservative, thus classical computations give the associated numerical fluxes,

$$
\begin{aligned}
& \mathcal{F}_{L}=\left(\mathcal{F}^{\rho}, \mathcal{F}^{\rho u_{1}}, \mathcal{F}^{\rho u_{2}}, \mathcal{F}_{L}^{\overline{\mathcal{E}_{e}}}, \mathcal{F}^{\overline{\mathcal{E}_{i}}}, \mathcal{F}^{B_{3}}\right), \\
& \mathcal{F}_{R}=\left(\mathcal{F}^{\rho}, \mathcal{F}^{\rho u_{1}}, \mathcal{F}^{\rho u_{2}}, \mathcal{F}_{R}^{\overline{\mathcal{E}}_{e}}, \mathcal{F}_{R}^{\overline{\mathcal{E}}_{i}}, \mathcal{F}^{B_{3}}\right),
\end{aligned}
$$

where the conservative part involves the Riemann solution evaluated at $\mathrm{x} / \mathrm{t}=0$,

$$
\begin{aligned}
& \mathcal{F}^{\rho}=(\rho u)_{x / t=0}, \\
& \mathcal{F}^{\rho u_{1}}=\left(\rho u_{1}^{2}+\pi_{e}+\pi_{i}+B_{3}^{2} / 2\right)_{x / t=0}, \\
& \mathcal{F}^{\rho u_{2}}=\left(\rho u_{1} u_{2}\right)_{x / t=0}, \\
& \mathcal{F}^{B_{3}}=\left(u_{1} B_{3}\right)_{x / t=0} .
\end{aligned}
$$

More explicitly (3.29) yields that the quantities between parentheses are evaluated at " $L$ " if $\Sigma_{1} \geq 0$, at " $L^{*}$ " if $\Sigma_{1} \leq 0 \leq \Sigma_{2}$, at " $R^{*}$ " if $\Sigma_{2} \leq 0 \leq \Sigma_{3}$, at " $R$ " if $\Sigma_{3} \leq 0$ (see Figure 1). As usual there is no ambiguity when equality occurs in these conditions.

We complete these formulas by computing the left/right numerical fluxes from (3.14) for the variables $\overline{\varepsilon_{\alpha}}$ with $\alpha=e, i$,

$$
\begin{aligned}
\mathcal{F}_{L}^{\overline{\varepsilon_{\alpha}}}= & \left(u_{1}\left(\overline{\varepsilon_{\alpha}}+\pi_{\alpha}+c_{\alpha} B_{3}^{2} / 2\right)\right)_{L}+\min \left(0, \Sigma_{1}\right)\left(\bar{\varepsilon}_{\alpha, L}^{*}-\bar{\varepsilon}_{\alpha, L}\right) \\
& +\min \left(0, \Sigma_{2}\right)\left(\bar{\varepsilon}_{\alpha, R}^{*}-\bar{\varepsilon}_{\alpha, L}^{*}\right)+\min \left(0, \Sigma_{3}\right)\left(\bar{\varepsilon}_{\alpha, R}-\bar{\varepsilon}_{\alpha, R}^{*}\right), \\
\mathcal{F}_{R}^{\overline{\varepsilon_{\alpha}}}= & \left(u_{1}\left(\overline{\varepsilon_{\alpha}}+\pi_{\alpha}+c_{\alpha} B_{3}^{2} / 2\right)\right)_{R}-\max \left(0, \Sigma_{1}\right)\left(\bar{\varepsilon}_{\alpha, L}^{*}-\bar{\varepsilon}_{\alpha, L}\right) \\
& -\max \left(0, \Sigma_{2}\right)\left(\bar{\varepsilon}_{\alpha, R}^{*}-\bar{\varepsilon}_{\alpha, L}^{*}\right)-\max \left(0, \Sigma_{3}\right)\left(\bar{\varepsilon}_{\alpha, R}-\bar{\varepsilon}_{\alpha, R}^{*}\right) .
\end{aligned}
$$




\subsubsection{Positivity of density and internal energies}

We must now provide some sufficient conditions on $a_{L}$ and $a_{R}$ in order to satisfy (3.21) and the realisability of the intermediate states, that is the positivity of $\rho_{L}^{*}, \rho_{R}^{*}, \varepsilon_{e, L}^{*}, \varepsilon_{i, L}^{*}, \varepsilon_{e, R}^{*}$ and $\varepsilon_{i, R}^{*}$. First, using (3.25), (3.26), (3.27), we obtain that

$$
\begin{aligned}
& a_{L}\left(a_{L}+a_{R}\right) \geq \rho_{L}\left(a_{R}\left(u_{1, L}-u_{1, R}\right)+\pi_{R}-\pi_{L}\right), \\
& a_{R}\left(a_{L}+a_{R}\right) \geq \rho_{R}\left(a_{L}\left(u_{1, L}-u_{1, R}\right)+\pi_{L}-\pi_{R}\right),
\end{aligned}
$$

are sufficient conditions to obtain (3.22) and thus are sufficient conditions to preserve the positivity of $\rho_{L}^{*}$ and $\rho_{R}^{*}$.

Second, from a straightforward calculation using (3.26), $\varepsilon_{e, L}^{*}, \varepsilon_{i, L}^{*}, \varepsilon_{e, R}^{*}$ and $\varepsilon_{i, R}^{*}$ are positive if

$$
\begin{aligned}
& a_{L} \geq \max \left(\frac{\left|\pi_{e, L}+c_{e} B_{3, L}^{2} / 2\right|}{2 c_{e} \sqrt{\varepsilon}_{e, L}}, \frac{\left|\pi_{i, L}+c_{i} B_{3, L}^{2} / 2\right|}{2 c_{i} \sqrt{\varepsilon}_{i, L}}\right), \\
& a_{R} \geq \max \left(\frac{\left|\pi_{e, R}+c_{e} B_{3, R}^{2} / 2\right|}{2 c_{e} \sqrt{\varepsilon}_{e, R}}, \frac{\left|\pi_{i, R}+c_{i} B_{3, R}^{2} / 2\right|}{2 c_{i} \sqrt{\varepsilon}_{i, R}}\right) .
\end{aligned}
$$

\section{Entropy minimum principle}

The present section is devoted to prove that the scheme (3.13) - (3.15) satisfies the following discrete entropy inequality

$$
\eta\left(U_{i}^{n+1}\right)-\eta\left(U_{i}^{n}\right)+\frac{\Delta t}{\Delta x}\left(G\left(U_{i}, U_{i+1}\right)-G\left(U_{i-1}, U_{i}\right)\right) \leq 0
$$

where

$$
\eta(U)=-\rho\left(s_{e}(U)+s_{i}(U)\right),
$$

is the entropy from (1.8). The numerical entropy flux $G\left(U_{l}, U_{r}\right)$ satisfies the consistency condition $G(U, U)=-\rho u\left(s_{e}(U)+s_{i}(U)\right)$.

The state vector $U$ is defined by (1.10) and belongs to the admissible state space $\Omega$ defined as follows

$$
\Omega=\left\{U \in \mathbb{R}^{6} ; \quad \rho>0, \quad \varepsilon_{e}>0, \quad \varepsilon_{i}>0\right\} .
$$

First we recall the classical and most general condition from Harten-Lax-van Leer [21] concerning discrete entropy inequalities. Let $\eta(U)$ be the entropy defined by (4.2). Let $R\left(\xi, U_{L}, U_{R}\right)$ be the approximate Riemann solver defined in Section 3.1.1. Under CFL condition (3.12), assume the following entropy consistency condition:

$$
\begin{aligned}
\frac{1}{\Delta x} \int_{-\Delta x / 2}^{\Delta x / 2} \eta\left(R\left(x / \Delta t, U_{L}, U_{R}\right)\right) d x & \leq \\
& \frac{1}{2}\left(\eta\left(U_{L}\right)+\eta\left(U_{R}\right)\right)-\frac{\Delta t}{\Delta x}\left(\eta\left(U_{R}\right) u_{R}+\eta\left(U_{L}\right) u_{L}\right) .
\end{aligned}
$$

Then the scheme (3.13) - (3.15) satisfies the discrete entropy inequality (4.1). We skip the proof of this well-know result (for instance, see [21]). 
The previous result states a criterion which is too weak to be applied on our scheme. Instead we use a stronger condition, based on a local minimum entropy principle. This idea was introduced in [9] for the gas dynamic sytem. A suitable extension of this technique has been derived for the MHD system in [11] and in [8] for the ten-moments system.

The first step consists in stating a sufficient condition on the intermediate states of the approximate Riemann solver to enforce the required discrete entropy inequality.

Theorem 4.1. Assume the intermediate states $U_{L}^{*}$ and $U_{R}^{*}$ defined by belong $\Omega$ for all $U_{L, R} \in \Omega$ and satisfy the following entropy estimates for $\alpha=e, i$

$$
s_{\alpha}\left(U_{L}^{*}\right) \geq s_{\alpha, L}, \quad s_{\alpha}\left(U_{R}^{*}\right) \geq s_{\alpha, R},
$$

where

$$
s_{\alpha, L}=s_{\alpha}\left(U_{L}\right), \quad s_{\alpha, R}=s_{\alpha}\left(U_{R}\right),
$$

and $s_{\alpha}(U)$ is given by (1.9). In addition assume the CFL condition (3.12) holds. Let $U_{i}^{n} \in \Omega$ for all $i \in \mathbb{Z}$. Then $U_{i}^{n+1}$ defined by (3.13)-(3.14) satisfies the discrete entropy inequality (4.1).

Remark 4.2. The result remains valid when $B_{3}=0$ and proves that the scheme developed by a Suliciu relaxation approach in [2] also satisfies a discrete entropy inequality.

Proof. Arguing similarly to the proof of the Theorem 6.1 in [8], the scheme will be proved to satisfy a discrete entropy inequality as soon as we have the following entropy estimates

$$
\eta\left(U_{L}^{*}\right) \geq \eta\left(U_{L}\right), \quad \eta\left(U_{R}^{*}\right) \geq \eta\left(U_{R}\right),
$$

and the following relations are satisfied

$$
\begin{aligned}
& \rho_{L}^{*} u_{1}^{*}-\rho_{L} u_{1, L}=\Sigma_{1}\left(\rho_{L}^{*}-\rho_{L}\right), \\
& \rho_{R}^{*} u_{1}^{*}-\rho_{R} u_{1, R}=\Sigma_{3}\left(\rho_{R}^{*}-\rho_{R}\right) .
\end{aligned}
$$

Using (4.2), we sum (4.4) for $\alpha=e, i$ and we obtain the entropy estimate. Moreover, these previous relations are Rankine-Hugoniot relations which are satisfied because the solver resolves exactly the equation on density (3.1).

Our goal is now to obtain the sufficient condition (4.4). In the sequel, we denote $\Sigma=\left(\lambda, \varepsilon_{\alpha}, B_{3}, \pi\right)^{T} \in \mathbb{R}^{+} \times \mathbb{R}^{+} \times \mathbb{R} \times \mathbb{R}$ and we set $\Sigma^{e q}=\left(\lambda, \varepsilon_{\alpha}, B_{3}, p_{\alpha}\left(\lambda, \varepsilon_{\alpha}\right)\right)^{T}$. Moreover we introduce three functions $\Sigma \mapsto \phi(\Sigma), \Sigma \mapsto \varphi(\Sigma), \Sigma \mapsto \psi(\Sigma)$ in $C^{2}\left(\mathbb{R}^{+} \times \mathbb{R}^{+} \times \mathbb{R} \times \mathbb{R}\right)$ associated to the Riemann invariants already exhibited in $(3.23)$, as follows

$$
\begin{aligned}
& \phi(\Sigma)=\pi+c_{\alpha} B_{3}^{2} / 2+c_{\alpha} a^{2} \lambda, \\
& \varphi(\Sigma)=\varepsilon_{\alpha}+\lambda B_{3}^{2}-\frac{\left(\pi+c_{\alpha} B_{3}^{2} / 2\right)^{2}}{2\left(c_{e} a\right)^{2}}, \\
& \psi(\Sigma)=\lambda B_{3},
\end{aligned}
$$

where we have set $\lambda$ the specific volume as follows

$$
\lambda=\frac{1}{\rho} .
$$

We now give our central technical statement. 
Proposition 4.3. Let $U$ defined by (1.10). Assume that (3.21) holds for all $U \in \Omega$. Then there exist a function $\Sigma \mapsto \mathcal{S}_{\alpha}(\phi(\Sigma), \varphi(\Sigma), \psi(\Sigma))$ so that

$$
\max _{\pi \in \mathbb{R}} \mathcal{S}_{\alpha}(\phi(\Sigma), \varphi(\Sigma), \psi(\Sigma))=\left.\mathcal{S}_{\alpha}(\phi(\Sigma), \varphi(\Sigma), \psi(\Sigma))\right|_{\pi=p_{\alpha}\left(\lambda, \varepsilon_{\alpha}\right)}=s_{\alpha}\left(\lambda, \varepsilon_{\alpha}\right) .
$$

Before we give the proof of this result, let us illustrate the interest of this technical proposition, by proving Theorem 4.1.

Proof of Theorem 4.1. Here we focus on the first inequality of (4.4) since the establishment of the second inequality is similarly obtained. First we apply Proposition 4.3. Thus there exists a function $\Sigma \mapsto \mathcal{S}_{\alpha}(\Sigma)$ such that relation (4.6) holds. As a consequence, if we denote

$$
\mathcal{S}_{\alpha}^{*}(\pi)=\mathcal{S}_{\alpha}^{*}\left(\phi\left(\lambda_{L}^{*}, \varepsilon_{\alpha, L}^{*}, B_{3, L}^{*}, \pi\right), \varphi\left(\lambda_{L}^{*}, \varepsilon_{\alpha, L}^{*}, B_{3, L}^{*}, \pi\right), \psi\left(\lambda_{L}^{*}, \varepsilon_{\alpha, L}^{*}, B_{3, L}^{*}, \pi\right)\right)
$$

then we have

$$
s_{\alpha}\left(\lambda_{L}^{*}, \varepsilon_{\alpha, L}^{*}\right)=\max _{\pi \in \mathbb{R}} \mathcal{S}_{\alpha}^{*}(\pi) \geq \mathcal{S}_{\alpha}^{*}(\widetilde{\pi}), \quad \forall \widetilde{\pi} \in \mathbb{R} .
$$

We fix $\widetilde{\pi}=\pi_{\alpha, L}^{*}$ to write

$$
s_{\alpha}\left(\lambda_{L}^{*}, \varepsilon_{\alpha, L}^{*}\right) \geq \mathcal{S}_{\alpha}^{*}\left(\pi_{L}^{*}\right) .
$$

Next the functions $\phi, \varphi, \psi$ defined by (4.5) are Riemann invariants shared by the eigenvalues $u_{1} \pm a / \rho$. Thus they are invariant across the wave speeds $u_{1, L}-a_{L} / \rho_{L}$ and $u_{1, R}+a_{R} / \rho_{R}$. So we have

$$
\begin{aligned}
& \varphi\left(\lambda_{L}^{*}, \varepsilon_{\alpha, L}^{*}, B_{3, L}^{*}, \pi_{\alpha, L}^{*}\right)=\varphi\left(\lambda_{L}, \varepsilon_{\alpha, L}, B_{3, L}, \pi_{\alpha, L}\right), \\
& \phi\left(\lambda_{L}^{*}, \varepsilon_{\alpha, L}^{*}, B_{3, L}^{*}, \pi_{\alpha, L}^{*}\right)=\phi\left(\lambda_{L}, \varepsilon_{\alpha, L}, B_{3, L}, \pi_{\alpha, L}\right) \\
& \psi\left(\lambda_{L}^{*}, \varepsilon_{\alpha, L}^{*}, B_{3, L}^{*}, \pi_{\alpha, L}^{*}\right)=\psi\left(\lambda_{L}, \varepsilon_{\alpha, L}, B_{3, L}, \pi_{\alpha, L}\right) .
\end{aligned}
$$

Last three equations imply that

$$
\begin{aligned}
& \mathcal{S}_{\alpha}^{*}\left(\pi_{L}^{*}\right) \\
& =\mathcal{S}_{\alpha}\left(\phi\left(\lambda_{L}, \varepsilon_{\alpha, L}, B_{3, L}, p_{L}\right), \varphi\left(\lambda_{L}, \varepsilon_{\alpha, L}, B_{3, L}, p_{L}\right), \psi\left(\lambda_{L}, \varepsilon_{\alpha, L}, B_{3, L}, p_{L}\right)\right) .
\end{aligned}
$$

Using last equality in (4.7) we get

$$
\begin{aligned}
& s_{\alpha}\left(\lambda_{L}^{*}, \varepsilon_{\alpha, L}^{*}\right) \\
& \geq \mathcal{S}_{\alpha}\left(\phi\left(\lambda_{L}, \varepsilon_{\alpha, L}, B_{3, L}, p_{L}\right), \varphi\left(\lambda_{L}, \varepsilon_{\alpha, L}, B_{3, L}, p_{L}\right), \psi\left(\lambda_{L}, \varepsilon_{\alpha, L}, B_{3, L}, p_{L}\right)\right) .
\end{aligned}
$$

In addition, using second equation in (4.6), we have

$$
\begin{aligned}
& s_{\alpha}\left(\lambda_{L}, \varepsilon_{\alpha, L}\right) \\
& =\mathcal{S}_{\alpha}\left(\phi\left(\lambda_{L}, \varepsilon_{\alpha, L}, B_{3, L}, p_{L}\right), \varphi\left(\lambda_{L}, \varepsilon_{\alpha, L}, B_{3, L}, p_{L}\right), \psi\left(\lambda_{L}, \varepsilon_{\alpha, L}, B_{3, L}, p_{L}\right)\right) .
\end{aligned}
$$

Thus the expected left minimum principle is reached and the proof is complete.

To complete this section, we now establish Proposition 4.3. To address such an issue, we need the following lemma whose helpfulness is just technical. In the sequel we set $\sigma=(\lambda, \varepsilon)^{T}$. 
Lemma 4.4. Let $U$ defined by (1.10) and $\phi(\Sigma), \varphi(\Sigma), \psi(\Sigma)$ by (4.5). Assume that (3.21) holds for all $U \in \Omega$. Then there exists three functions, denoted by $\bar{\lambda}(X, Y, Z), \bar{\varepsilon}_{\alpha}(X, Y, Z), \bar{B}_{3}(X, Y, Z)$ such that

$$
\begin{aligned}
\bar{\lambda}\left(\phi\left(\Sigma^{e q}\right), \varphi\left(\Sigma^{e q}\right), \psi\left(\Sigma^{e q}\right)\right)=\lambda, & \bar{\varepsilon}_{\alpha}\left(\phi\left(\Sigma^{e q}\right), \varphi\left(\Sigma^{e q}\right), \psi\left(\Sigma^{e q}\right)\right)=\varepsilon \\
\text { and } \quad & \bar{B}_{3}\left(\phi\left(\Sigma^{e q}\right), \varphi\left(\Sigma^{e q}\right), \psi\left(\Sigma^{e q}\right)\right)=B_{3},
\end{aligned}
$$

and the following derivatives are satisfied:

$$
\begin{aligned}
& \frac{\partial \bar{\lambda}}{\partial X}(X, Y, Z)=\frac{1}{D(\tilde{\sigma})}\left(\bar{\lambda}-\frac{\gamma-1}{c_{\alpha} a^{2}}\left(p_{\alpha}\left(\bar{\lambda}, \bar{\varepsilon}_{\alpha}\right)+c_{\alpha} \frac{\bar{B}_{3}^{2}}{2}\right)\right) \\
& \frac{\partial \bar{\lambda}}{\partial Y}(X, Y, Z)=\frac{-c_{\alpha}(\gamma-1)}{D(\tilde{\sigma})} \\
& \frac{\partial \bar{\varepsilon}_{\alpha}}{\partial X}(X, Y, Z)=\frac{1}{D(\tilde{\sigma})}\left(\lambda \frac{\bar{B}_{3}^{2}}{2}-\frac{p_{\alpha}\left(\bar{\lambda}, \bar{\varepsilon}_{\alpha}\right)+c_{\alpha} \bar{B}_{3}^{2}}{\left(c_{\alpha} a\right)^{2}}\left(p_{\alpha}\left(\bar{\lambda}, \bar{\varepsilon}_{\alpha}\right)+c_{\alpha} \frac{\bar{B}_{3}^{2}}{2}\right)\right) \\
& \frac{\partial \bar{\varepsilon}_{\alpha}}{\partial Y}(X, Y, Z)=\frac{1}{D(\tilde{\sigma})}\left(\bar{\lambda} a^{2}-p_{\alpha}\left(\bar{\lambda}, \bar{\varepsilon}_{\alpha}\right)-c_{\alpha} \bar{B}_{3}^{2}\right) \\
& \frac{\partial \bar{B}_{3}}{\partial X}(X, Y, Z)=\frac{-\bar{B}_{3}}{D(\tilde{\sigma})}\left(1-\frac{\gamma-1}{\bar{\lambda} c_{\alpha} a^{2}}\left(p_{\alpha}\left(\bar{\lambda}, \bar{\varepsilon}_{\alpha}\right)+c_{\alpha} \frac{\bar{B}_{3}^{2}}{2}\right)\right) \\
& \frac{\partial \bar{B}_{3}}{\partial Y}(X, Y, Z)=\frac{c_{\alpha}(\gamma-1) \bar{B}_{3}}{\bar{\lambda} D(\tilde{\sigma})}
\end{aligned}
$$

where we have set

$$
\tilde{\sigma}=\left(\bar{\lambda}(X, Y, Z), \bar{\varepsilon}_{\alpha}(X, Y, Z), \bar{B}_{3}(X, Y, Z)\right)^{T}
$$

and

$$
D\left(\lambda, \varepsilon_{\alpha}, B_{3}\right)=c_{\alpha} \lambda a^{2}-\gamma_{\alpha} p_{\alpha}\left(\lambda, \varepsilon_{\alpha}\right)-c_{\alpha} B_{3}^{2} .
$$

In the second statement, we exhibit a suitable function derived from $\bar{\lambda}$ and $\bar{\varepsilon}$ and we consider its local extremum.

Lemma 4.5. Let $U$ defined by (1.10) and $\phi(\Sigma), \varphi(\Sigma), \psi(\Sigma)$ by (4.5). Assume that (3.21) holds for all $U \in \Omega$. We introduce $S(\Sigma): \mathbb{R}^{+} \times \mathbb{R}^{+} \times \mathbb{R} \times \mathbb{R} \rightarrow \mathbb{R}$ defined by:

$$
S(\Sigma)=s_{\alpha}\left(\bar{\lambda}(\phi(\Sigma), \varphi(\Sigma), \psi(\Sigma)), \bar{\varepsilon}_{\alpha}(\phi(\Sigma), \varphi(\Sigma), \psi(\Sigma))\right),
$$

where $s_{\alpha}\left(\lambda, \varepsilon_{\alpha}\right)$ denotes the specific entropy. For sake of clarity we denote $\bar{\lambda}, \bar{\varepsilon}_{\alpha}$ and $\bar{B}_{3}$ instead of $\bar{\lambda}(\varphi(\Sigma), \phi(\Sigma), \psi(\Sigma)), \bar{\varepsilon}_{\alpha}(\varphi(\Sigma), \phi(\Sigma), \psi(\Sigma))$, and $\bar{B}_{3}(\varphi(\Sigma), \phi(\Sigma), \psi(\Sigma))$. Then we have

$$
\frac{\partial S}{\partial \pi}(\Sigma)=\frac{\left(p_{\alpha}\left(\bar{\lambda}, \bar{\varepsilon}_{\alpha}\right)+\bar{B}_{3}^{2} / 2\right)-\left(\pi+B_{3}^{2} / 2\right)}{a^{2} \bar{\varepsilon}_{\alpha}},
$$

with the functions $\bar{\lambda}, \bar{\varepsilon}_{\alpha}$ and $\bar{B}_{3}$ defined in Lemma 4.4.

The last result concerns the study of the extrema of the function $S$ defined by (4.17). 
Lemma 4.6. Let $U$ defined by (1.10) and $S(\Sigma)$ defined by (4.17). Assume that (3.21) holds for all $U \in \Omega$. Then the function $\pi \mapsto S(\Sigma)$ admits a unique maximum given by $\pi=p_{\alpha}\left(\lambda, \varepsilon_{\alpha}\right)$.

Equipped with these results, we can establish Proposition 4.3

Proof of Proposition 4.3. From assumption (3.21), we can apply Lemmas 4.4 and 4.5 to define a function $\mathcal{S}$ as follows:

$$
\begin{aligned}
\mathcal{S}(\phi(\Sigma), \varphi(\Sigma), \psi(\Sigma)) & =\mathcal{S}(\Sigma), \\
& =s\left(\bar{\lambda}(\phi(\Sigma), \varphi(\Sigma), \psi(\Sigma)), \bar{\varepsilon}_{\alpha}(\phi(\Sigma), \varphi(\Sigma), \psi(\Sigma))\right),
\end{aligned}
$$

where the function $s$ is nothing but the specific entropy. Now, by definition of $\bar{\lambda}$ and $\bar{\varepsilon}_{\alpha}$, we have

$$
\left.\bar{\lambda}(\phi(\Sigma), \varphi(\Sigma), \psi(\Sigma))\right|_{\pi=p\left(\lambda, \varepsilon_{\alpha}\right)}=\lambda \text { and }\left.\bar{\varepsilon}_{\alpha}(\phi(\Sigma), \varphi(\Sigma), \psi(\Sigma))\right|_{\pi=p\left(\lambda, \varepsilon_{\alpha}\right)}=\varepsilon_{\alpha},
$$

which immediately implies

$$
\left.\mathcal{S}(\phi(\Sigma), \varphi(\Sigma), \psi(\Sigma))\right|_{\pi=p\left(\lambda, \varepsilon_{\alpha}\right)}=s\left(\lambda, \varepsilon_{\alpha}\right) .
$$

Next, we study the extrema of the function $\pi \mapsto S(\Sigma)$. By Lemma 4.6, the unique maximum of the function $S$ is $\pi=p_{\alpha}\left(\lambda, \varepsilon_{\alpha}\right)$, which completes the proof.

We conclude this section by giving successively the proof of the three intermediate lemmas.

Proof of Lemma 4.4. Let us consider the function

$$
\Theta:\left(\lambda, \varepsilon_{\alpha}, B_{3}\right) \mapsto\left(\begin{array}{c}
\phi\left(\lambda, \varepsilon_{\alpha}, B_{3}, p_{\alpha}\left(\lambda, \varepsilon_{\alpha}\right)\right) \\
\varphi\left(\lambda, \varepsilon_{\alpha}, B_{3}, p_{\alpha}\left(\lambda, \varepsilon_{\alpha}\right)\right) \\
\psi\left(\lambda, \varepsilon_{\alpha}, B_{3}, p_{\alpha}\left(\lambda, \varepsilon_{\alpha}\right)\right)
\end{array}\right) .
$$

We remark that the function $D\left(\lambda, \varepsilon_{\alpha}, B_{3}\right)$, defined by (4.16) is nothing but the Jacobian function of $\Theta$. Since for all $\left(\lambda, \varepsilon_{\alpha}, B_{3}\right)$ under consideration (3.21) holds, $D\left(\lambda, \varepsilon_{\alpha}, B_{3}\right)$ does not vanish. Thus we can apply the inverse function theorem to deduce the existence of a reciprocal function

$$
\Theta^{-1}(X, Y, Z)=\left(\begin{array}{l}
\bar{\lambda}(X, Y, Z) \\
\bar{\varepsilon}_{\alpha}(X, Y, Z) \\
\bar{B}_{3}(X, Y, Z)
\end{array}\right)
$$

defined for $(X, Y, Z)$ in the range of $\Theta$ and such that $D\left(\lambda, \varepsilon_{\alpha}, B_{3}\right) \neq 0$.

By definition of the functions $\bar{\lambda}, \bar{\varepsilon}_{\alpha}$ and $\bar{B}_{3}$, the relation (4.9) is obviously obtained.

Now, we evaluate the derivatives of those reciprocal functions. Once again by definition of $\bar{\lambda}, \bar{\varepsilon}_{\alpha}$ and $\bar{B}_{3}$, we have

$$
\begin{aligned}
& \varphi\left(\bar{\lambda}(X, Y, Z), \bar{B}_{3}(X, Y, Z), p_{\alpha}\left(\bar{\lambda}, \bar{\varepsilon}_{\alpha}\right)\right)=X, \\
& \phi\left(\bar{\lambda}(X, Y, Z), \bar{\varepsilon}_{\alpha}(X, Y, Z), \bar{B}_{3}(X, Y, Z), p_{\alpha}\left(\bar{\lambda}, \bar{\varepsilon}_{\alpha}\right)\right)=Y, \\
& \psi\left(\bar{\lambda}(X, Y, Z), \bar{B}_{3}(X, Y, Z)\right)=Z .
\end{aligned}
$$


By differentiating in $X$ these two relations and using (4.5), we obtain:

$$
\begin{aligned}
\left(c_{\alpha} a^{2}-\frac{p_{\alpha}\left(\bar{\lambda}, \bar{\varepsilon}_{\alpha}\right)}{\bar{\lambda}}\right) \frac{\partial \bar{\lambda}}{\partial X}+\frac{c_{\alpha}(\gamma-1)}{\bar{\lambda}} \frac{\partial \bar{\varepsilon}_{\alpha}}{\partial X}+c_{\alpha} \bar{B}_{3} \frac{\partial \bar{B}_{3}}{\partial X} & =1 \\
\left(\frac{\bar{B}_{3}^{2}}{2}+\frac{p_{\alpha}\left(\bar{\lambda}, \bar{\varepsilon}_{\alpha}\right)}{\left(c_{\alpha} a\right)^{2} \bar{\lambda}}\left(p_{\alpha}\left(\bar{\lambda}, \bar{\varepsilon}_{\alpha}\right)+c_{\alpha} \frac{\bar{B}_{3}^{2}}{2}\right)\right) \frac{\partial \bar{\lambda}}{\partial X} & \\
+\left(1-\frac{p_{\alpha}\left(\bar{\lambda}, \bar{\varepsilon}_{\alpha}\right)}{\left(c_{\alpha} a\right)^{2} \bar{\varepsilon}_{\alpha}}\left(p_{\alpha}\left(\bar{\lambda}, \bar{\varepsilon}_{\alpha}\right)+c_{\alpha} \frac{\bar{B}_{3}^{2}}{2}\right)\right) \frac{\partial \bar{\varepsilon}_{\alpha}}{\partial X} & =0 \\
+\bar{B}_{3}\left(\bar{\lambda}-\frac{1}{c_{\alpha} a^{2}}\left(p_{\alpha}\left(\bar{\lambda}, \bar{\varepsilon}_{\alpha}\right)+c_{\alpha} \frac{\bar{B}_{3}^{2}}{2}\right)\right) \frac{\partial \bar{B}_{3}}{\partial X} & =0 .
\end{aligned}
$$

With $D\left(\lambda, \varepsilon_{\alpha}, B_{3}\right) \neq 0$, this above $3 \times 3$ system is solvable in the variables $\left(\frac{\partial \bar{\lambda}}{\partial X}, \frac{\partial \bar{\varepsilon}_{\alpha}}{\partial X}, \frac{\partial \bar{B}_{3}}{\partial X}\right)$. Then we get the expected definition of the derivatives $\frac{\partial \bar{\lambda}}{\partial X}(X, Y, Z)$, $\frac{\partial \bar{\varepsilon}_{\alpha}}{\partial X}(X, Y, Z)$ and $\frac{\partial \bar{B}_{3}}{\partial X}(X, Y, Z)$ given by (4.10), (4.12), and (4.14).

Proof of Lemma 4.5. Here, we have to compute the derivative of $S$ with respect to $\pi$ where the function $S$ is defined by (4.17). Using the definition of $\psi$ in (4.5) we notice that $\frac{\partial \psi}{\partial \pi}(\Sigma)=0$, which enables us to write

$$
\begin{array}{r}
\frac{\partial S}{\partial \pi}(\Sigma)=\frac{\partial s_{\alpha}}{\partial \lambda}(\bar{\sigma})\left(\frac{\partial \bar{\lambda}}{\partial X}(\varphi(\Sigma), \phi(\Sigma), \psi(\Sigma)) \frac{\partial \varphi}{\partial \pi}(\Sigma)\right. \\
\left.+\frac{\partial \bar{\lambda}}{\partial Y}(\varphi(\Sigma), \phi(\Sigma), \psi(\Sigma)) \frac{\partial \phi}{\partial \pi}(\Sigma)\right) \\
+\frac{\partial s_{\alpha}}{\partial \varepsilon_{\alpha}}(\bar{\sigma})\left(\frac{\partial \bar{\varepsilon}_{\alpha}}{\partial X}(\varphi(\Sigma), \phi(\Sigma), \psi(\Sigma)) \frac{\partial \varphi}{\partial \pi}(\Sigma)\right. \\
\left.+\frac{\partial \bar{\varepsilon}_{\alpha}}{\partial Y}(\varphi(\Sigma), \phi(\Sigma), \psi(\Sigma)) \frac{\partial \phi}{\partial \pi}(\Sigma)\right),
\end{array}
$$

where we have set

$$
\bar{\sigma}=\left(\bar{\lambda}(\varphi(\Sigma), \phi(\Sigma), \psi(\Sigma)), \bar{\varepsilon}_{\alpha}(\varphi(\Sigma), \phi(\Sigma), \psi(\Sigma))\right)^{T} .
$$

In the sequel, we will denote $\bar{\lambda}, \bar{\varepsilon}_{\alpha}$ and $\bar{B}_{3}$ instead of $\bar{\lambda}(\varphi(\Sigma), \phi(\Sigma), \psi(\Sigma))$, $\bar{\varepsilon}_{\alpha}(\varphi(\Sigma), \phi(\Sigma), \psi(\Sigma))$ and $\bar{B}_{3}(\varphi(\Sigma), \phi(\Sigma), \psi(\Sigma))$. Moreover we will use the notation $\tilde{\sigma}=\left(\bar{\lambda}, \bar{\varepsilon}_{\alpha}, \bar{B}_{3}\right)$. Next using (4.10)-(4.13), we compute

$$
\begin{gathered}
\frac{\partial \bar{\lambda}}{\partial X}(\varphi(\Sigma), \phi(\Sigma), \psi(\Sigma)) \frac{\partial \varphi}{\partial \pi}(\Sigma)+\frac{\partial \bar{\lambda}}{\partial Y}(\varphi(\Sigma), \phi(\Sigma), \psi(\Sigma)) \frac{\partial \phi}{\partial \pi}(\Sigma)= \\
\frac{1}{D(\tilde{\sigma})}\left(\bar{\lambda}+\frac{(\gamma-1)}{c_{\alpha} a^{2}}\left[\left(\pi+c_{\alpha} \frac{B_{3}^{2}}{2}\right)-\left(p_{\alpha}(\bar{\lambda}, \bar{\varepsilon})+c_{\alpha} \frac{\bar{B}_{3}^{2}}{2}\right)\right]\right),
\end{gathered}
$$

and

$$
\begin{array}{r}
\frac{\partial \bar{\varepsilon}_{\alpha}}{\partial X}(\varphi(\Sigma), \phi(\Sigma), \psi(\Sigma)) \frac{\partial \varphi}{\partial \pi}(\Sigma)+\frac{\partial \bar{\varepsilon}_{\alpha}}{\partial Y}(\varphi(\Sigma), \phi(\Sigma), \psi(\Sigma)) \frac{\partial \phi}{\partial \pi}(\Sigma)= \\
\frac{1}{D(\tilde{\sigma})}\left(\bar{\lambda}\left(\frac{\bar{B}_{3}^{2}}{2}-\frac{1}{c_{\alpha}}\left(\pi+c_{\alpha} \frac{B_{3}^{2}}{2}\right)\right)+\frac{p_{\alpha}\left(\bar{\lambda}, \bar{\varepsilon}_{\alpha}\right)+c_{\alpha} \bar{B}_{3}^{2}}{\left(c_{\alpha} a\right)^{2}}\right. \\
\left.\left[\left(\pi+c_{\alpha} \frac{B_{3}^{2}}{2}\right)-\left(p_{\alpha}(\bar{\lambda}, \bar{\varepsilon})+c_{\alpha} \frac{\bar{B}_{3}^{2}}{2}\right)\right]\right) .
\end{array}
$$


Then we plug into (4.20) the two previous relations together with

$$
\frac{\partial s_{\alpha}}{\partial \lambda}(\bar{\sigma})=\frac{(\gamma-1)}{\bar{\lambda}}, \quad \text { and } \quad \frac{\partial s_{\alpha}}{\partial \varepsilon_{\alpha}}(\bar{\sigma})=\frac{1}{\varepsilon_{\alpha}},
$$

to obtain the following equation:

$$
\frac{\partial S}{\partial \pi}(\Sigma)=\frac{1}{D(\tilde{\sigma})}\left(A+B\left[\left(\pi+c_{\alpha} \frac{B_{3}^{2}}{2}\right)-\left(p_{\alpha}(\bar{\lambda}, \bar{\varepsilon})+c_{\alpha} \frac{\bar{B}_{3}^{2}}{2}\right)\right]\right),
$$

with

$$
A=(\gamma-1)+\frac{\bar{\lambda}}{c_{\alpha} \bar{\varepsilon}_{\alpha}}\left(c_{\alpha} \frac{\bar{B}_{3}^{2}}{2}-\pi-c_{\alpha} \frac{B_{3}^{2}}{2}\right), B=\frac{(\gamma-1)^{2}}{c_{\alpha} a^{2} \bar{\lambda}}+\frac{p_{\alpha}(\bar{\lambda}, \bar{\varepsilon})+c_{\alpha} \bar{B}_{3}^{2}}{\left(c_{\alpha} a\right)^{2} \bar{\varepsilon}_{\alpha}} .
$$

We now write

$$
A=\frac{\bar{\lambda}}{c_{\alpha} \bar{\varepsilon}_{\alpha}}\left(p_{\alpha}\left(\bar{\lambda}, \bar{\varepsilon}_{\alpha}\right)+c_{\alpha} \frac{\bar{B}_{3}^{2}}{2}-\pi-c_{\alpha} \frac{B_{3}^{2}}{2}\right), \quad B=\frac{\gamma p_{\alpha}\left(\bar{\lambda}, \bar{\varepsilon}_{\alpha}\right)+c_{\alpha} \bar{B}_{3}^{2}}{\left(c_{\alpha} a\right)^{2} \bar{\varepsilon}_{\alpha} \bar{\lambda}} .
$$

Finally we plug these equalities into (4.23) which gives (4.18).

Proof of Lemma 4.6. We apply Lemma 4.4 and Lemma 4.5, which enables us to introduce the function

$$
g(\Sigma)=\left(p_{\alpha}\left(\bar{\lambda}, \bar{\varepsilon}_{\alpha}\right)+\bar{B}_{3}^{2} / 2\right) \circ(\phi(\Sigma), \varphi(\Sigma), \psi(\Sigma))-\left(\pi+B_{3}^{2} / 2\right),
$$

where $\Sigma=\left(\lambda, \varepsilon_{\alpha}, B_{3}, \pi\right)^{T}$.

In order to prove the lemma, we are going to study the roots of the function $g$. First we notice that, thanks to relations in (4.9), $\pi^{0}=p_{\alpha}\left(\lambda, \varepsilon_{\alpha}\right)$ is a root of $g$. Then we will show that for every $\tilde{\pi}^{0}$ root of $g$, we have $\frac{\partial g}{\partial \pi}\left(\tilde{\pi}^{0}\right)<0$.

Let $\tilde{\pi}^{0}$ be a root of $g$. We denote by $g^{\prime}$ the partial derivative of $g$ with respect to $\pi$, and we compute it as follows:

$$
\begin{aligned}
g^{\prime}(\Sigma)= & \frac{\partial p_{\alpha}}{\partial \lambda}(\bar{\sigma})\left(\frac{\partial \bar{\lambda}}{\partial X}(\varphi(\Sigma), \phi(\Sigma), \psi(\Sigma)) \frac{\partial \varphi}{\partial \pi}(\Sigma)\right. \\
& \left.+\frac{\partial \bar{\lambda}}{\partial Y}(\varphi(\Sigma), \phi(\Sigma), \psi(\Sigma)) \frac{\partial \phi}{\partial \pi}(\Sigma)\right) \\
+ & \frac{\partial p_{\alpha}}{\partial \varepsilon_{\alpha}}(\bar{\sigma})\left(\frac{\partial \bar{\varepsilon}_{\alpha}}{\partial X}(\varphi(\Sigma), \phi(\Sigma), \psi(\Sigma)) \frac{\partial \varphi}{\partial \pi}(\Sigma)\right. \\
& \left.+\frac{\partial \bar{\varepsilon}_{\alpha}}{\partial Y}(\varphi(\Sigma), \phi(\Sigma), \psi(\Sigma)) \frac{\partial \phi}{\partial \pi}(\Sigma)\right) \\
+ & \bar{B}_{3}(\varphi(\Sigma), \phi(\Sigma), \psi(\Sigma))\left(\frac{\partial \bar{B}_{3}}{\partial X}(\varphi(\Sigma), \phi(\Sigma), \psi(\Sigma)) \frac{\partial \varphi}{\partial \pi}(\Sigma)\right. \\
& \left.+\frac{\partial \bar{B}_{3}}{\partial Y}(\varphi(\Sigma), \phi(\Sigma), \psi(\Sigma)) \frac{\partial \phi}{\partial \pi}(\Sigma)\right)-1,
\end{aligned}
$$

where

$$
\bar{\sigma}=\left(\bar{\lambda}(\varphi(\Sigma), \phi(\Sigma), \psi(\Sigma)), \bar{\varepsilon}_{\alpha}(\varphi(\Sigma), \phi(\Sigma), \psi(\Sigma))\right)^{T} .
$$

In the sequel, we use the notation $\Sigma^{0}=\left(\lambda, \varepsilon_{\alpha}, B_{3}, \tilde{\pi}^{0}\right)$ and we are interested in computing $g^{\prime}\left(\Sigma^{0}\right)$. For sake of clarity we will denote $\bar{\lambda}, \bar{\varepsilon}_{\alpha}$ and $\bar{B}_{3}$ instead of $\bar{\lambda}\left(\varphi\left(\Sigma^{0}\right), \phi\left(\Sigma^{0}\right), \psi\left(\Sigma^{0}\right)\right), \bar{\varepsilon}_{\alpha}\left(\varphi\left(\Sigma^{0}\right), \phi\left(\Sigma^{0}\right), \psi\left(\Sigma^{0}\right)\right)$ and $\bar{B}_{3}\left(\varphi\left(\Sigma^{0}\right), \phi\left(\Sigma^{0}\right), \psi\left(\Sigma^{0}\right)\right)$. 
Moreover we will denote $\left.\tilde{\sigma}=\left(\bar{\lambda}, \bar{\varepsilon}_{\alpha}, \bar{B}_{3}\right)\right)^{T}$.

First we use the previous computations (4.21), (4.22) and the fact that $g\left(\Sigma^{0}\right)=0$ to get

$$
\begin{aligned}
\frac{\partial \bar{\lambda}}{\partial X}\left(\varphi\left(\Sigma^{0}\right), \phi\left(\Sigma^{0}\right), \psi\left(\Sigma^{0}\right)\right) \frac{\partial \varphi}{\partial \pi} & \left(\Sigma^{0}\right) \\
& +\frac{\partial \bar{\lambda}}{\partial Y}\left(\varphi\left(\Sigma^{0}\right), \phi\left(\Sigma^{0}\right), \psi\left(\Sigma^{0}\right)\right) \frac{\partial \phi}{\partial \pi}\left(\Sigma^{0}\right)=\frac{\bar{\lambda}}{D(\tilde{\sigma})},
\end{aligned}
$$

and

$$
\begin{aligned}
& \frac{\partial \bar{\varepsilon}_{\alpha}}{\partial X}\left(\varphi\left(\Sigma^{0}\right), \phi\left(\Sigma^{0}\right), \psi\left(\Sigma^{0}\right)\right) \frac{\partial \varphi}{\partial \pi}\left(\Sigma^{0}\right) \\
& +\frac{\partial \bar{\varepsilon}_{\alpha}}{\partial Y}\left(\varphi\left(\Sigma^{0}\right), \phi\left(\Sigma^{0}\right), \psi\left(\Sigma^{0}\right)\right) \frac{\partial \phi}{\partial \pi}\left(\Sigma^{0}\right)=\frac{-\bar{\lambda} p_{\alpha}\left(\bar{\lambda}, \bar{\varepsilon}_{\alpha}\right)}{c_{\alpha} D(\tilde{\sigma})} .
\end{aligned}
$$

Moreover combining $g\left(\Sigma^{0}\right)=0$ and relations (4.5), (4.14), (4.15) we get that

$$
\begin{aligned}
\frac{\partial \bar{B}_{3}}{\partial X}\left(\varphi\left(\Sigma^{0}\right), \phi\left(\Sigma^{0}\right), \psi\left(\Sigma^{0}\right)\right) & \frac{\partial \varphi}{\partial \pi}\left(\Sigma^{0}\right) \\
& +\frac{\partial \bar{B}_{3}}{\partial Y}\left(\varphi\left(\Sigma^{0}\right), \phi\left(\Sigma^{0}\right), \psi\left(\Sigma^{0}\right)\right) \frac{\partial \phi}{\partial \pi}\left(\Sigma^{0}\right)=\frac{-c_{\alpha} \bar{B}}{D(\tilde{\sigma})} .
\end{aligned}
$$

Then we plug in (4.24) last three previous relations together with

$$
\frac{\partial p_{\alpha}}{\partial \lambda}(\bar{\sigma})=\frac{-p_{\alpha}}{\lambda} \quad \text { and } \quad \frac{\partial s_{\alpha}}{\partial \varepsilon_{\alpha}}(\bar{\sigma})=\frac{p_{\alpha}}{\varepsilon_{\alpha}},
$$

in order to get

$$
g^{\prime}\left(\Sigma^{0}\right)=-\frac{\gamma p_{\alpha}\left(\bar{\lambda}, \bar{\varepsilon}_{\alpha}\right)+c_{\alpha} \bar{B}_{3}+D(\tilde{\sigma})}{D(\tilde{\sigma})} .
$$

Thus $g^{\prime}\left(\Sigma^{0}\right)<0$ for all roots $g$. The function $g$ being continuous, $\pi \mapsto g(\Sigma)$ has a unique root, which is $\pi=p_{\alpha}\left(\lambda, \varepsilon_{\alpha}\right)$.

To complete the proof we will show that $\pi=p_{\alpha}\left(\lambda, \varepsilon_{\alpha}\right)$ is a maximum of the function $S$ in Lemma 4.5. Indeed we can rewrite (4.18) as

$$
\frac{\partial S}{\partial \pi}(\Sigma)=\frac{g(\Sigma)}{a^{2} \bar{\varepsilon}_{\alpha}(\varphi(\Sigma), \phi(\Sigma), \psi(\Sigma))} .
$$

Since $\pi=p_{\alpha}\left(\lambda, \varepsilon_{\alpha}\right)$ is a unique root of $g, g\left(\Sigma^{e q}\right)=0$ and from the previous relation we deduce that $\frac{\partial S}{\partial \pi}\left(\Sigma^{e q}\right)=0$. Moreover we have

$$
\frac{\partial^{2} S}{\partial \pi^{2}}\left(\Sigma^{e q}\right)=\frac{g^{\prime}\left(\Sigma^{e q}\right)}{a^{2} \bar{\varepsilon}_{\alpha}\left(\varphi\left(\Sigma^{e q}\right), \phi\left(\Sigma^{e q}\right), \psi\left(\Sigma^{e q}\right)\right)},
$$

and we have previously shown that $g^{\prime}$ is negative for all roots of $g$. So we finally get that $\frac{\partial S}{\partial \pi}\left(\Sigma^{e q}\right)=0$ and $\frac{\partial^{2} S}{\partial \pi^{2}}\left(\Sigma^{e q}\right)<0$. Thus $\pi=p_{\alpha}\left(\lambda, \varepsilon_{\alpha}\right)$ is the maximum of the function $S$, which concludes the proof. 


\section{$5 \quad$ Numerical tests}

In this section we perform numerical approximations in order to evaluate both accuracy and robustness of the scheme. First order in time and space are evaluated. The CFL number is $1 / 2$ in all tests. The computations are performed over interval $[0,1]$. Boundary conditions are transmissive. For all test case we use the following values for the parameters

$$
k_{B}=1, \quad \gamma_{e}=\gamma_{i}=\frac{5}{3}, \quad Z=1, \quad m_{e}=10^{-3}, \quad m_{i}=1, \quad u_{2}=0 .
$$

The numerical test are organized as follows. The subsection 5.1 is dedicated to the approximation of a smooth analytical solution for the full system (the source term is a non-zero function). The subsection 5.2 is dedicated to the approximation of discontinuous solutions of the homogeneous system (parameters are taken such that the source term is the identically zero function).

\subsection{Source term approximation: an analytical solution}

We take initial data such that

$$
\forall x \in \mathbb{R}, \quad \rho(x, 0)=1, \quad u_{1}(x, 0)=10, \quad T_{i}(x, 0)+T_{e}(x, 0)=\bar{T}=2 .
$$

An exact solution of the system (1.1)-(1.6) is available,

$$
\begin{aligned}
& \forall(x, t) \in \mathbb{R} \times \mathbb{R}^{+}, \quad \rho(x, t)=1, \quad u_{1}(x, t)=10, \\
& \begin{aligned}
T_{e}(x, t)= & T_{e}\left(x-u_{1} t, 0\right) e^{-2 \mu t} \\
& +a(x, t)\left(1-e^{-2 \mu t}\right)+b(x, t)\left(2 \mu t-1+e^{-2 \mu t}\right),
\end{aligned}
\end{aligned}
$$

where $\mu=\left(\gamma_{\alpha}-1\right) \nu_{1, \alpha \beta} /\left(n_{e} k_{B}\right)$ and

$$
\begin{aligned}
& a(x, t)=\frac{\bar{T}}{2}+\frac{\widetilde{\nu}_{2, e i}}{2 \nu_{e i}^{1}}\left(\partial_{x} B_{3}\left(x-u_{1} t, 0\right)\right)^{2}, \\
& b(x, t)=\frac{\widetilde{\nu}_{2, e i}+\widetilde{\nu}_{2, i e}}{4 \mu}\left(\partial_{x} B_{3}\left(x-u_{1} t, 0\right)\right)^{2} .
\end{aligned}
$$

Here we choose $\bar{T}=2$ and for all $x \in[0,1]$,

$$
T_{e}(x, 0)=\frac{\bar{T}}{2}+\exp \left(-200(x-1 / 2)^{2}\right), \text { and } B_{3}(x, 0)=\exp \left(-50(x-1 / 2)^{2}\right) .
$$

Moreover we set $\nu_{1, e i}=1$ and $\widetilde{\nu}_{2, e i}=\widetilde{\nu}_{2, i e}$. The results for $\widetilde{\nu}_{2, e i}=2$ and $\widetilde{\nu}_{2, e i}=4$ are displayed in figure 2 . They show a good agreement with analytical solution (5.1).

\subsection{Resolution of the Riemann Problem}

In this section, we consider seven test cases that are inspired from the book of Toro ([29]) in order to highlight the robustness of the numerical method and take into account the magnetic field. In all chosen tests, data consists of two constant 

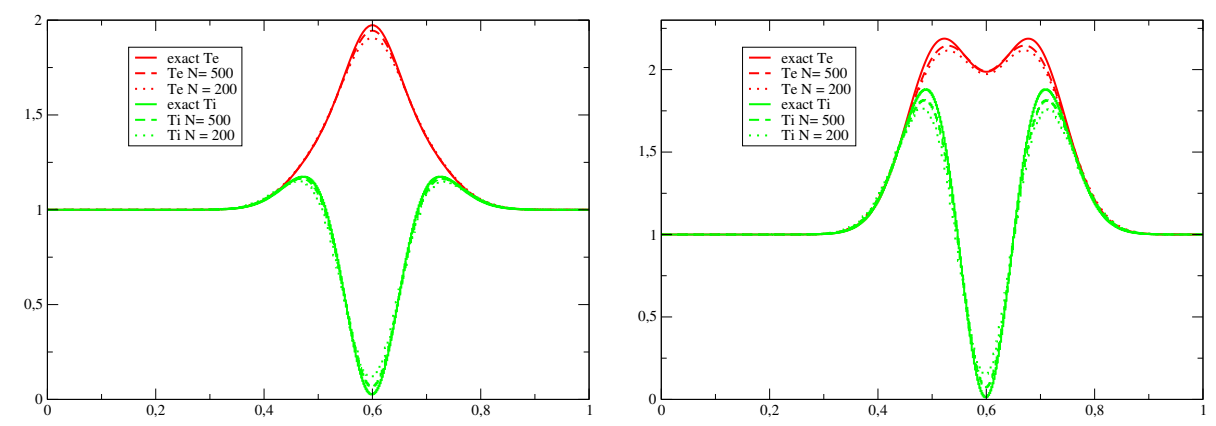

Figure 2: Solution for analytic test case for the temperatures $T_{e}, T_{i}$ computed with 200 and 500 cells. On the left $\widetilde{\nu}_{2, e i}=2$ and on the right $\widetilde{\nu}_{2, e i}=4$.

states $W_{L}=\left(\rho_{L}, u_{1, L}, T_{e, L}, T_{i, L}, B_{3, L}\right)^{T}$ and $W_{R}=\left(\rho_{R}, u_{1, R}, T_{e, R}, T_{i, R}, B_{3, R}\right)^{T}$, separated by a discontinuity at a position $x=x_{0}$. The states $W_{L}$ and $W_{R}$ are given in Table 1. These test cases are Riemann problems in the space domain $[0,1]$. For each test problem we select a convenient position $x_{0}$ of the initial discontinuity and the output time. These are stated in the legend of each figure displaying computational results.

\begin{tabular}{|l|l|l|l|l|l|}
\hline Test & $\rho$ & $u$ & $B_{3}$ & $T_{e}$ & $T_{i}$ \\
\hline Test 1 left & 1 & 0.75 & 0.8164966 & 0.3336667 & 0.3336667 \\
\hline Test 1 right & 0.125 & 0 & 0.2581989 & 0.2669333 & 0.2669333 \\
\hline Test 2 left & 1 & -2 & 0.5163978 & 0.1334667 & 0.1334667 \\
\hline Test 2 right & 1 & 2 & 0.5163978 & 0.1334667 & 0.1334667 \\
\hline Test 3 left & 1 & 0 & 14.142136 & 100.10000 & 100.1 \\
\hline Test 3 right & 1 & 0 & 0.2581989 & 0.0333667 & 0.0333667 \\
\hline Test 4 left & 5.9999924 & 19.5975 & 17.528909 & 25.630859 & 25.630860 \\
\hline Test 4 right & 5.9999242 & -6.19633 & 5.5434646 & 2.5634264 & 2.5634266 \\
\hline Test 5 left & 1 & -19.5975 & 8.1649658 & 33.366665 & 33.366667 \\
\hline Test 5 right & 1 & -19.5975 & 0.2581989 & 0.0333667 & 0.0333667 \\
\hline Test 6 left & 1.4 & 0 & 0.8164966 & 0.2383333 & 0.2383333 \\
\hline Test 6 right & 1 & 0 & 0.8164966 & 0.3336667 & 0.3336667 \\
\hline Test 7 left & 1.4 & 0.1 & 0.8164966 & 0.2383333 & 0.2383333 \\
\hline Test 7 right & 1 & 0.1 & 0.8164966 & 0.3336667 & 0.3336667 \\
\hline
\end{tabular}

Table 1: Data for seven test problems.

Test 1 is a modified Sod test case. The solution is constituted by a right shock wave, a right travelling contact and a left sonic rarefaction wave. Test case 2 consists of two symmetric rarefaction waves travelling in opposite direction and a trivial contact wave. The solution of Test 3 consists of a strong wave of shock, a contact surface and a left rarefaction wave. The solution of Test 4 is composed of three strong discontinuities travelling to the right. The solution of Test 5 corresponds to a left rarefaction rarefaction wave, a right-travelling shock wave and a stationary contact discontinuity. The solution of Test 6 is an 
isolated stationary contact wave. The solution of Test 7 is an isolated contact moving slowly to the right.

We compare our Suliciu method with a nonconservative version of the classical HLL method. Like the Sulicu method, it is a nonconservative finite volume type of scheme, with update formula (3.13). In the following we explain how we define the numerical fluxes $F_{l}\left(U_{l}, U_{r}\right)$ and $F_{r}\left(U_{l}, U_{r}\right)$. First we use HLL numerical fluxes (denoted by the subscript HLL in the following) to discretize the conservative part of our system. It leads to conservative fluxes $\left(F_{l}=F_{r}=F\right)$ for the conserved quantities $\rho, \rho u_{1}, \rho u_{2}, B_{3}$ :

$$
\begin{gathered}
F^{\rho}\left(U_{l}, U_{r}\right)=F_{\mathrm{HLL}}^{\rho}\left(U_{l}, U_{r}\right) \\
F^{\rho u_{1}}\left(U_{l}, U_{r}\right)=F_{\mathrm{HLL}}^{\rho u_{1}}\left(U_{l}, U_{r}\right) \\
F^{\rho u_{2}}\left(U_{l}, U_{r}\right)=F_{\mathrm{HLL}}^{\rho u_{2}}\left(U_{l}, U_{r}\right) \\
F^{B_{3}}\left(U_{l}, U_{r}\right)=F_{\mathrm{HLL}}^{B_{3}}\left(U_{l}, U_{r}\right) .
\end{gathered}
$$

In addition we use a centered discretisation of the nonconservative terms. In this subsection we will use the notation $\phi=c_{i} p_{e}-c_{e} p_{i}$ so that the nonconservative terms now write $-u_{1} \partial_{x} \phi$ in (1.4) and $u_{1} \partial_{x} \phi$ in (1.5) .This leads to nonconservative fluxes $\left(F_{l} \neq F_{r}\right)$ for the components $\overline{\varepsilon_{e}}$ and $\overline{\varepsilon_{i}}$ :

$$
\begin{gathered}
F_{l}^{\overline{\varepsilon_{e}}}\left(U_{l}, U_{r}\right)=F_{\mathrm{HLL}}^{\overline{\varepsilon_{e}}}\left(U_{l}, U_{r}\right)-u_{l}\left(\phi_{r}-\phi_{l}\right) \\
F_{r}^{\overline{\varepsilon_{e}}}\left(U_{l}, U_{r}\right)=F_{\mathrm{HLL}}^{\overline{\varepsilon_{e}}}\left(U_{l}, U_{r}\right)-u_{r}\left(\phi_{r}-\phi_{l}\right) \\
F_{l}^{\overline{\varepsilon_{i}}}\left(U_{l}, U_{r}\right)=F_{\mathrm{HLL}}^{\overline{\varepsilon_{e}}}\left(U_{l}, U_{r}\right)+u_{l}\left(\phi_{r}-\phi_{l}\right) \\
F_{r}^{\overline{\varepsilon_{i}}}\left(U_{l}, U_{r}\right)=F_{\mathrm{HLL}}^{\overline{\varepsilon_{e}}}\left(U_{l}, U_{r}\right)+u_{r}\left(\phi_{r}-\phi_{l}\right) .
\end{gathered}
$$

The nonservative HLL method is thus defined by (3.13), (5.2)-(5.9) with $\phi=$ $c_{i} p_{e}-c_{e} p_{i}$.

\subsubsection{Numerical results}

The results are presented as follows. Figure 3 to Figure 7 show results for Suliciu method. Figure 8 to Figure 10 show results for the nonconservative HLL method. Figure 11 show results aimed at comparing the performance of the nonconservative HLL and the Suliciu methods for isolated, stationnary and slowly moving contact discontinuities.

For Test 1 to Test 5 , in presence of magnetic field no exact solutions are available. Thus we compute numerical solutions for 300, 2000, 10000 cells. The quantities shown are density $\rho$, speed $u_{1}$, electronic and ionic temperatures $T_{e}$ and $T_{i}$, and magnetic field $B_{3}$. The goal is to investigate qualitative behavior of numerical methods. Figure 3 to Figure 7 show three good properties of the Suliciu method. First, the Suliciu method is able to produce solutions for all tests. Second it converges when the number of cell increases. Third all densities and temperatures computed remain positives. Figure 8 to Figure 10 show that the previous good properties of the Sulicu method are not met when not dealing properly with nonconservative products. First, the nonconservative HLL fails to produce solutions for Test 2 and Test 5 . On the other tests, the 
density remains positive but the ionic temperatures do not on Figure 8 and 10. Moreover, the convergence does not occur. More precisely, on Figure 8, for ionic temperature and magnetic field, a spike grows when the number of cell is increasing. For electronic temperature, unphysical oscillations occur. On Figure 9, a spike grows for density and magnetic field, unphysical oscillations grow on ionic temperature. On Figure 10 or a spike appears and grows for density, magnetic field and ionic temperature.

For Test 6 and Test 7, exact solutions are available. We compare numerical solution for 300 cells and the exact solution. Figure 11 shows a good property that the Suliciu method has and the nonconservative HLL has not. The Sulicu method is exact on isolated stationnary contact discontinuity, and very sharp on isolated slowly moving contact discontinuity. On the other hand, the nonconservative HLL spreads too much those discontinuities.

\section{Conclusion}

In this paper we have investigated at the modelling and numerical point of view the bitemperature Euler system with transverse magnetic field.

At the modelling point of view, we introduced a multicomponent BGK kinetic system coupled with Mawell equations. Next by perfoming an hydrodynamic limit, the bitemperature Euler equations with transverse magnetic field has been established.

At the numerical level, we designed a Suliciu relaxation approximation and we showed that the associated scheme is entropic. We compared our method with an a priori not positive nor entropic scheme, a nonconservative version of the classical HLL method. The results highlight the fundamental role of designing positive and entropic schemes to obtain their convergence.

We shall address to forthcoming papers the following different points. Firstly, we are currently working on taking into account transverse electric (TE) fields. Then, we plan to use this work dealing with transverse magnetic field and future work on TE field in order to propose novel high order and multi-dimensional schemes. Moreover, the case of Navier-Stokes asymptotics has also to be considered. 

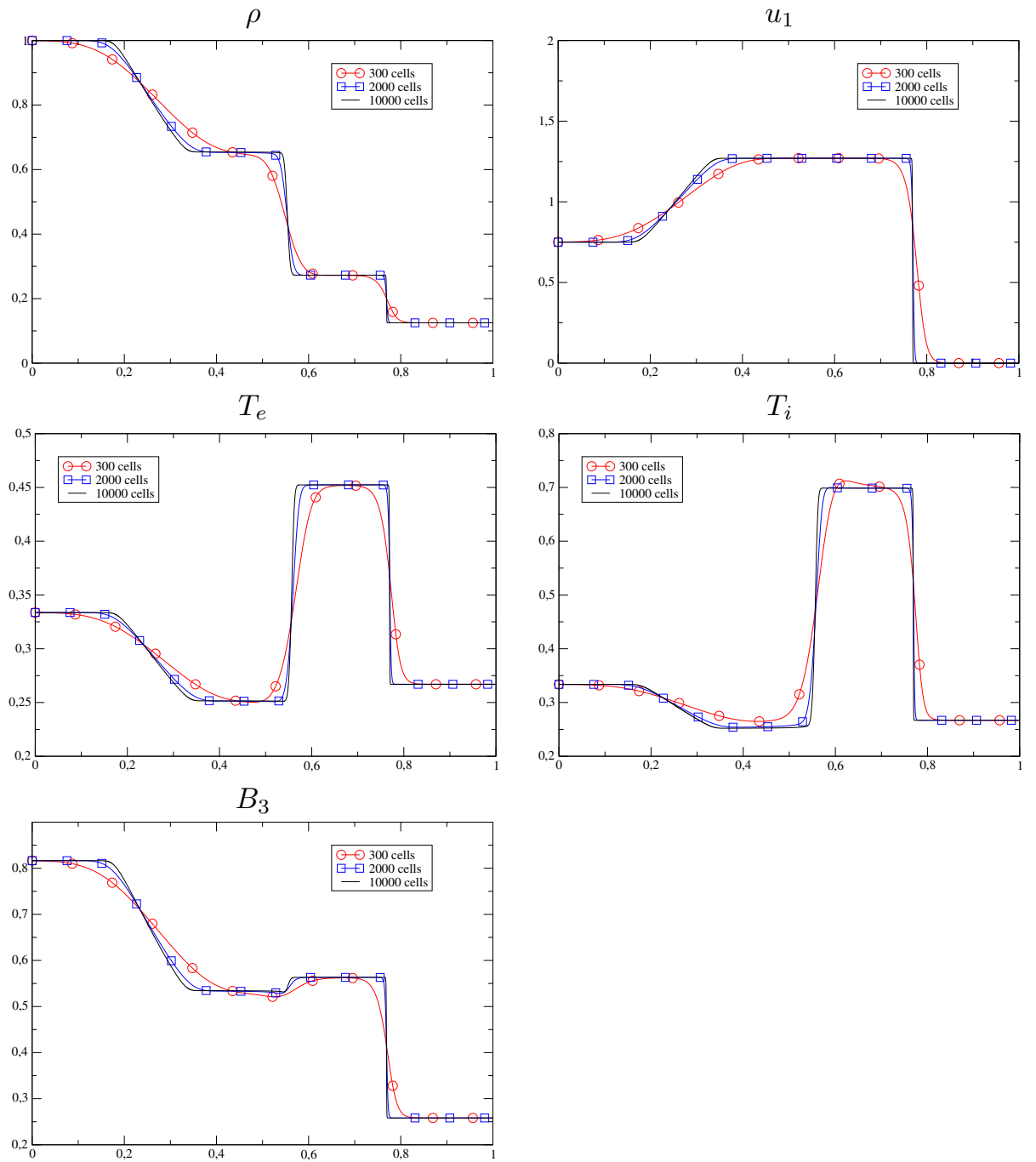

Figure 3: Suliciu method applied to Test 1 with $x_{0}=0.3$. Numerical solutions are compared at computational time $t=0.2$. 

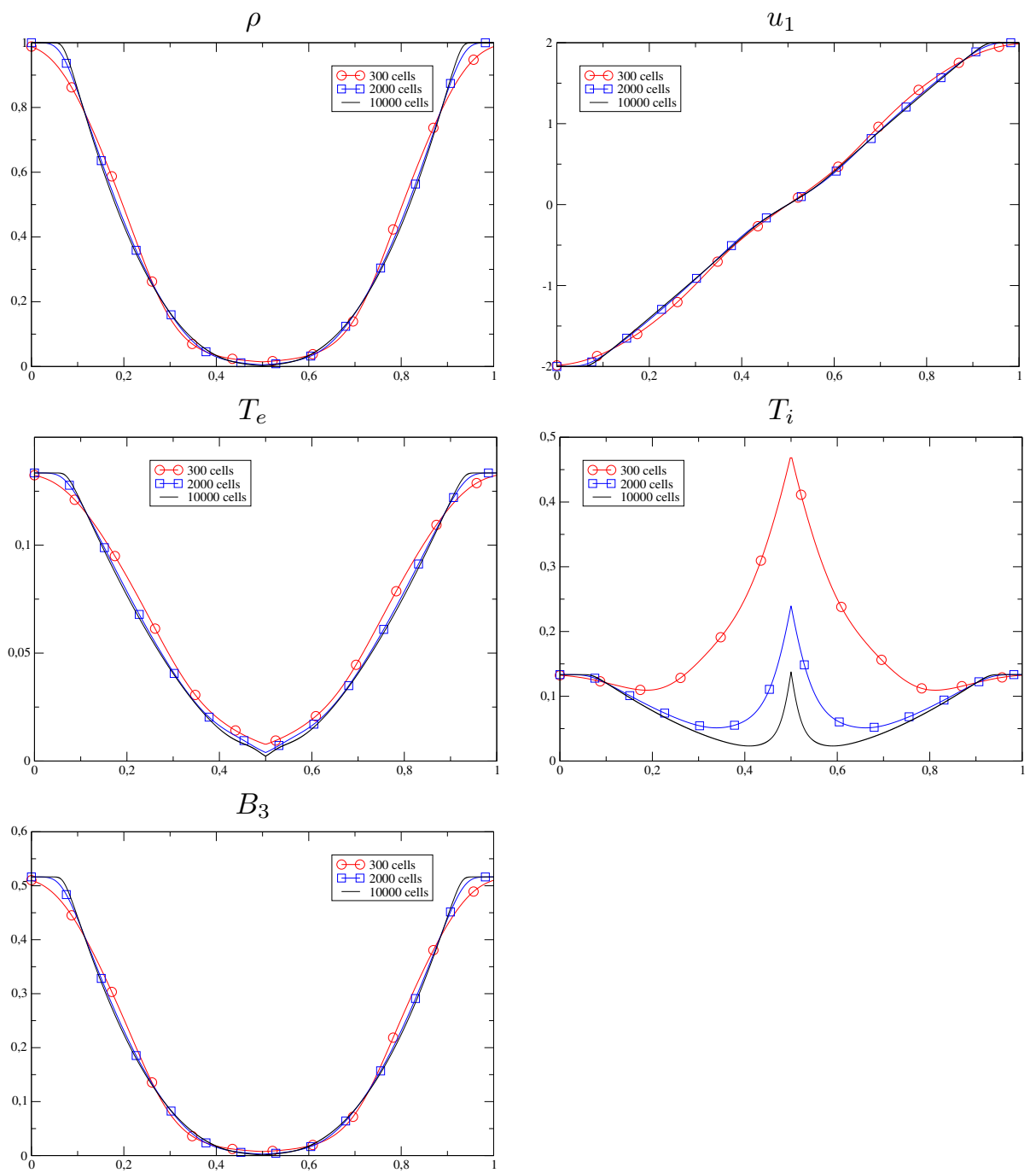

Figure 4: Suliciu method applied to Test 2 with $x_{0}=0.5$. Numerical solutions are compared at computational time $t=0.15$. 

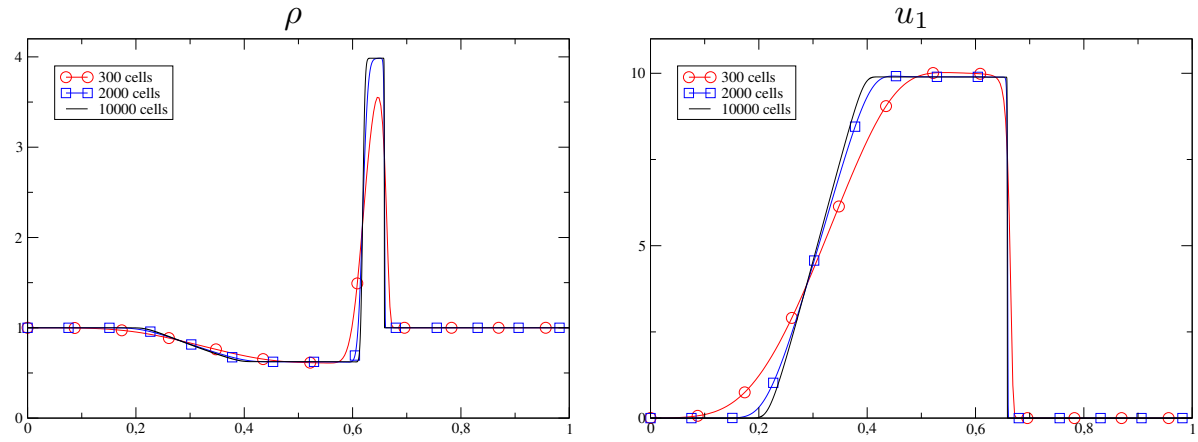

$T_{e}$
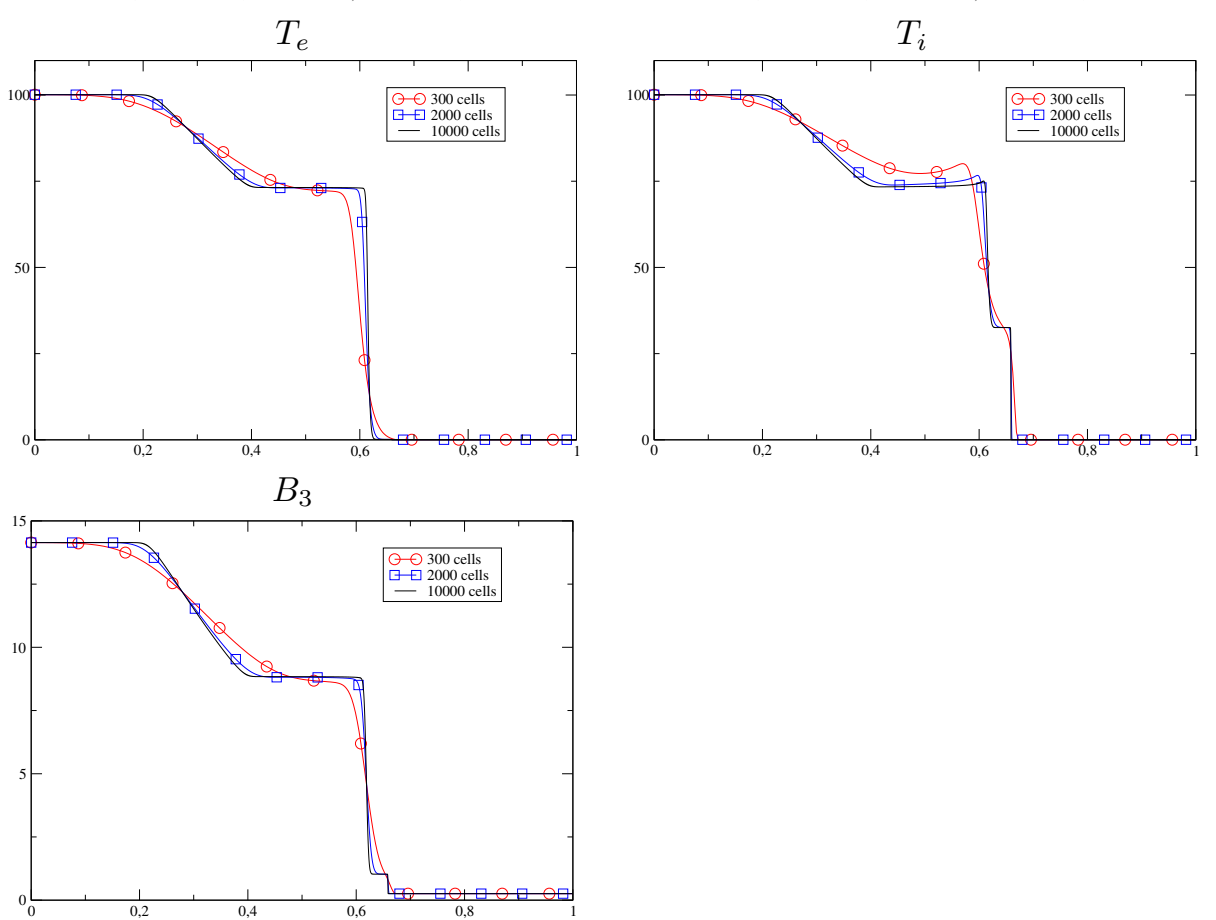

Figure 5: Suliciu method applied to Test 3 with $x_{0}=0.5$. Numerical solutions are compared at computational time $t=0.012$. 

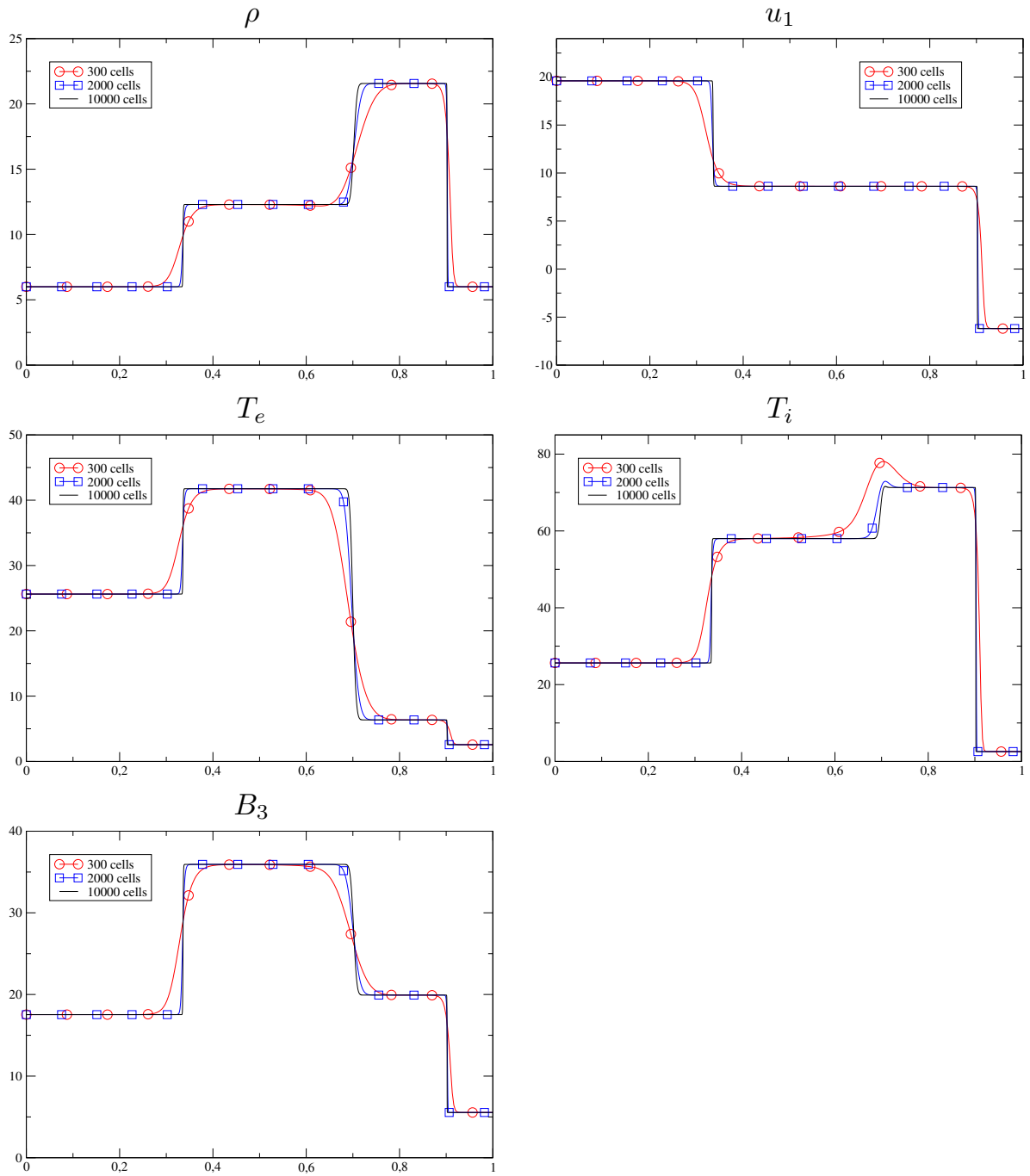

Figure 6: Suliciu method applied to Test 4 with $x_{0}=0.4$. Numerical solutions are compared at computational time $t=0.035$. 

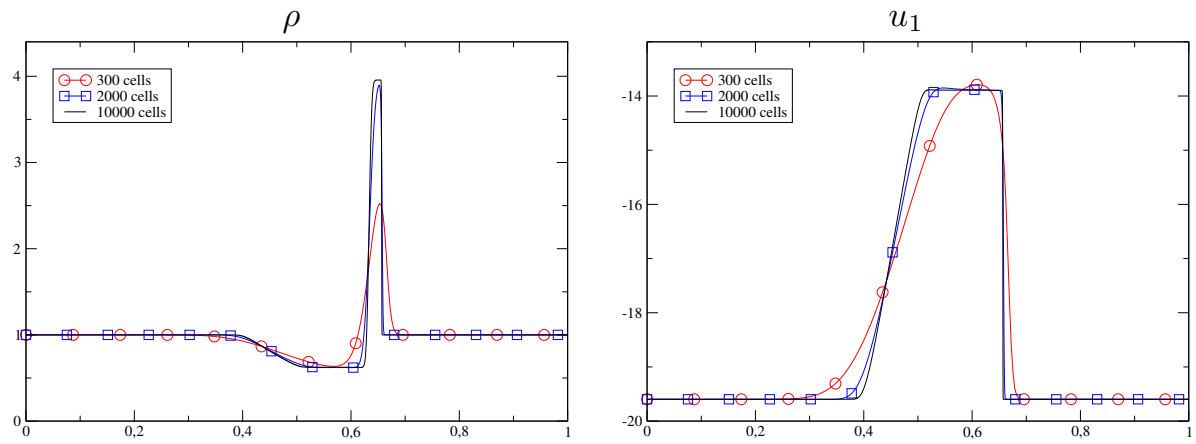

$T_{e}$
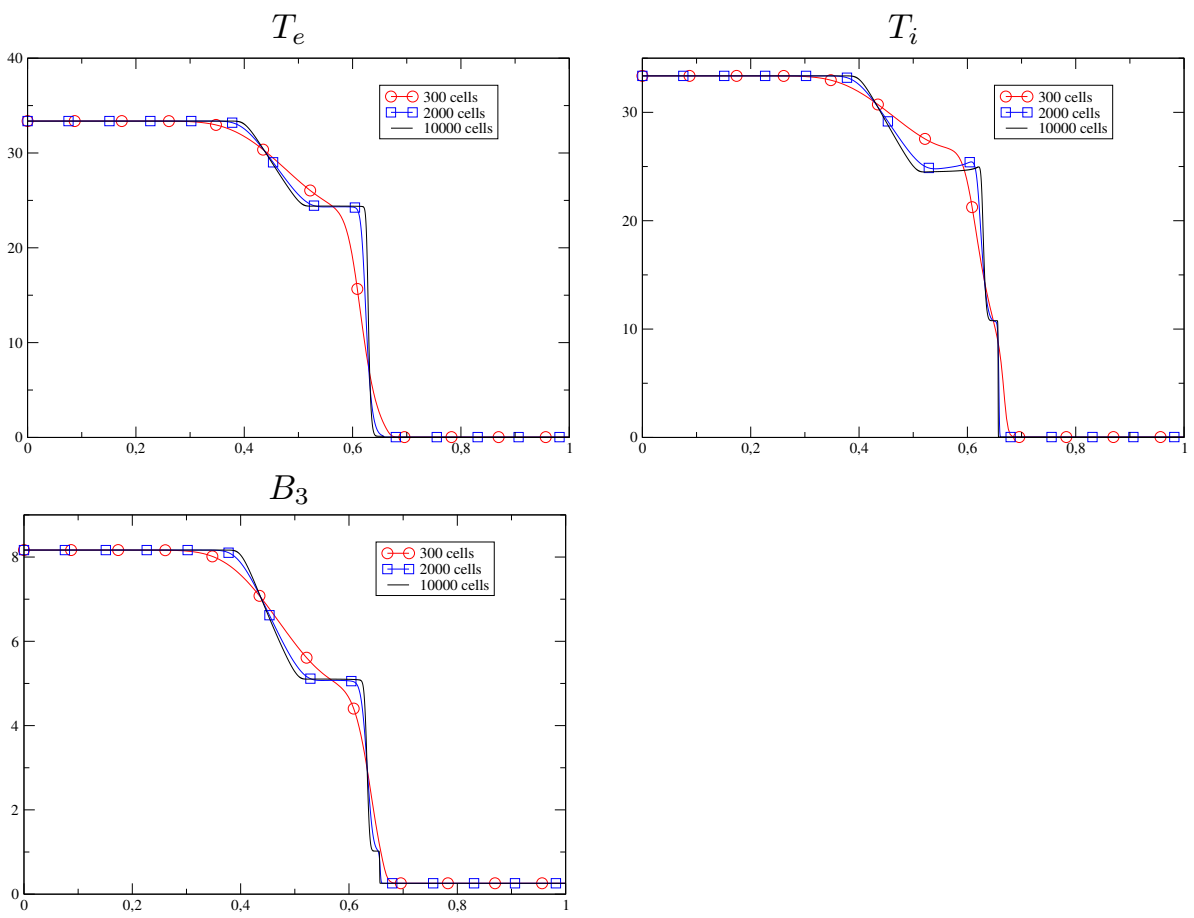

Figure 7: Suliciu method applied to Test 5 with $x_{0}=0.8$. Numerical solutions are compared at computational time $t=0.012$. 

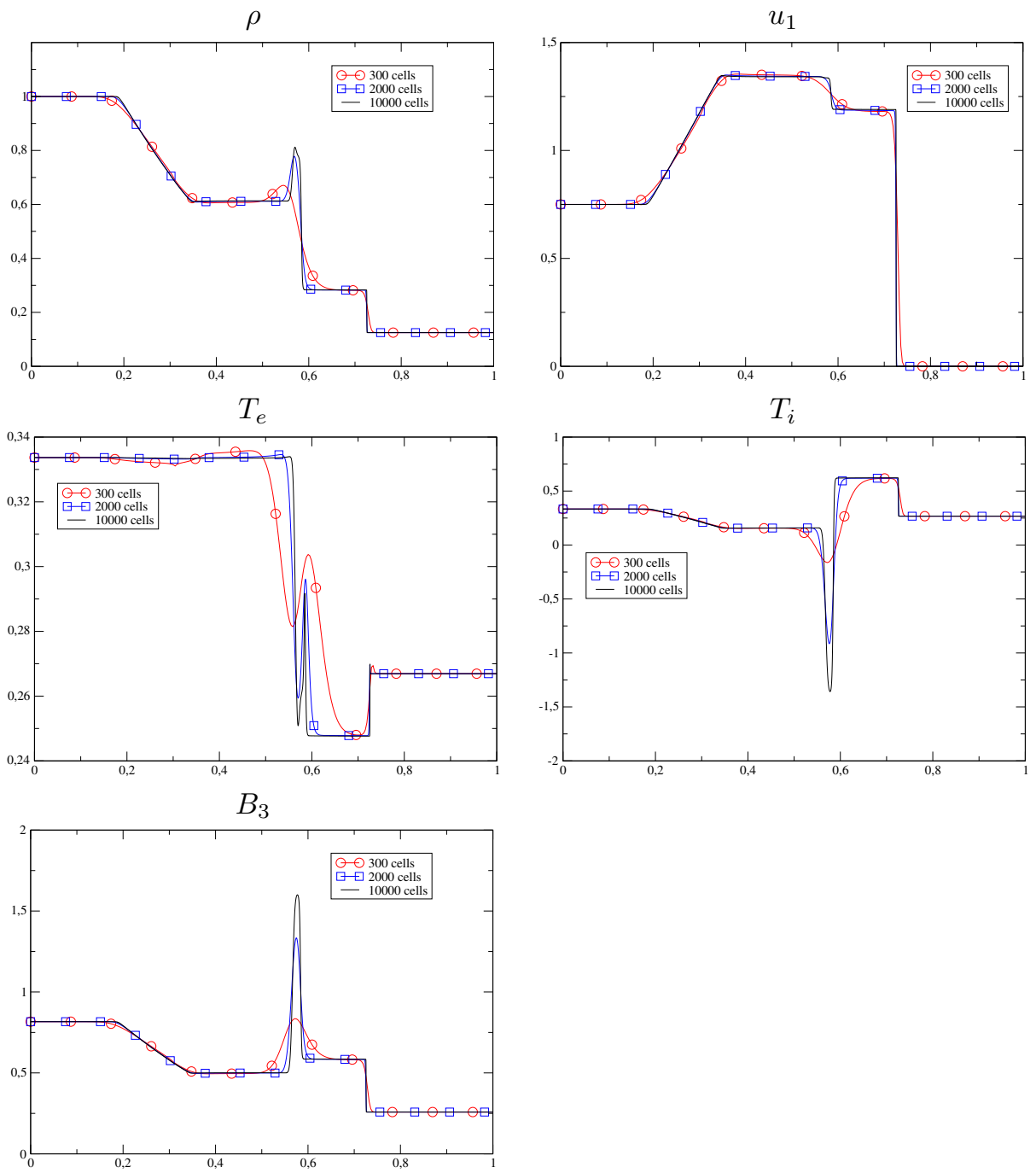

Figure 8: Nonconservative HLL method applied to Test 1 with $x_{0}=0.3$. Numerical solutions are compared at computational time $t=0.2$. 

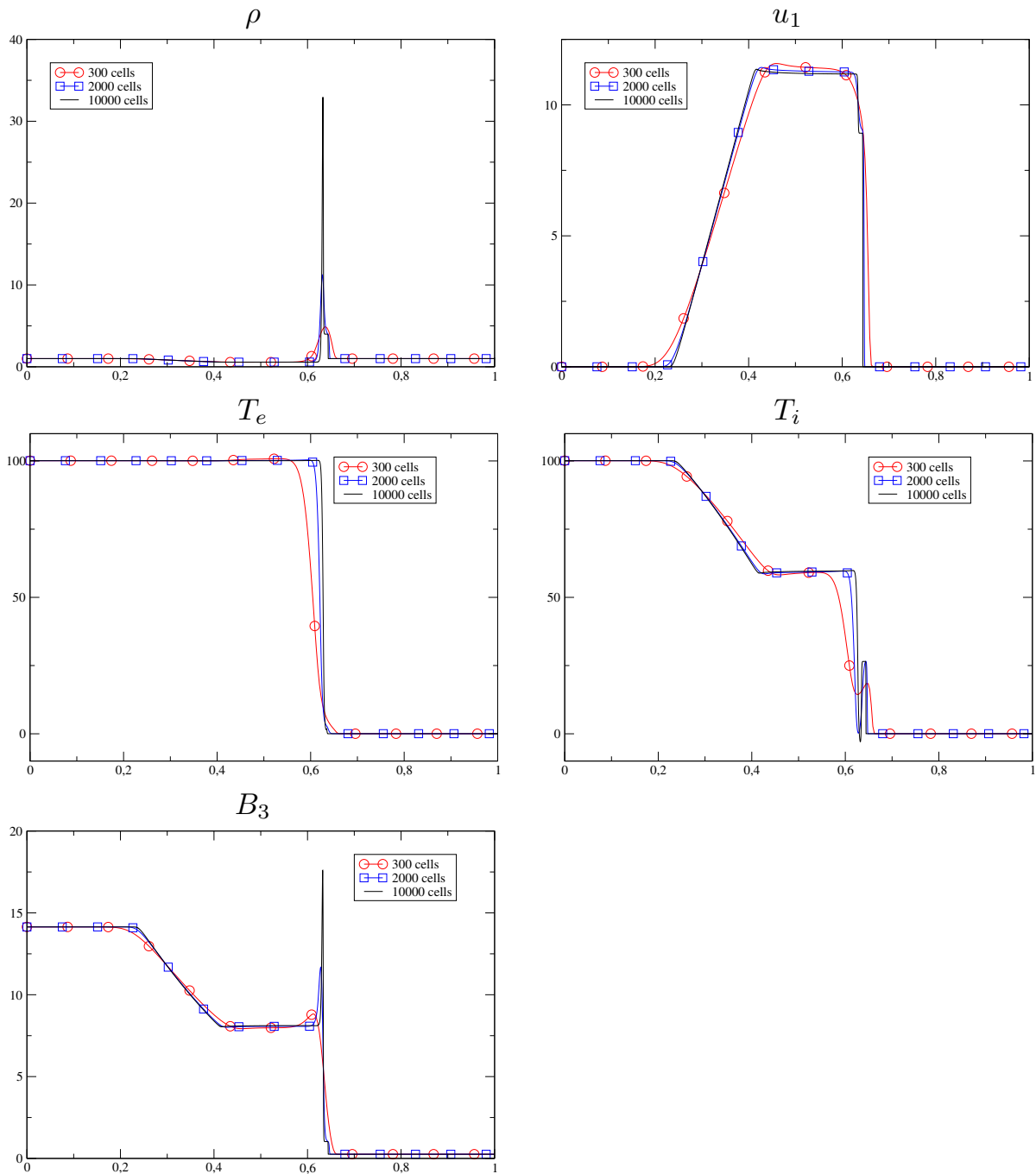

Figure 9: Nonconservative HLL method applied to Test 3 with $x_{0}=0.5$. Numerical solutions are compared at computational time $t=0.012$. 

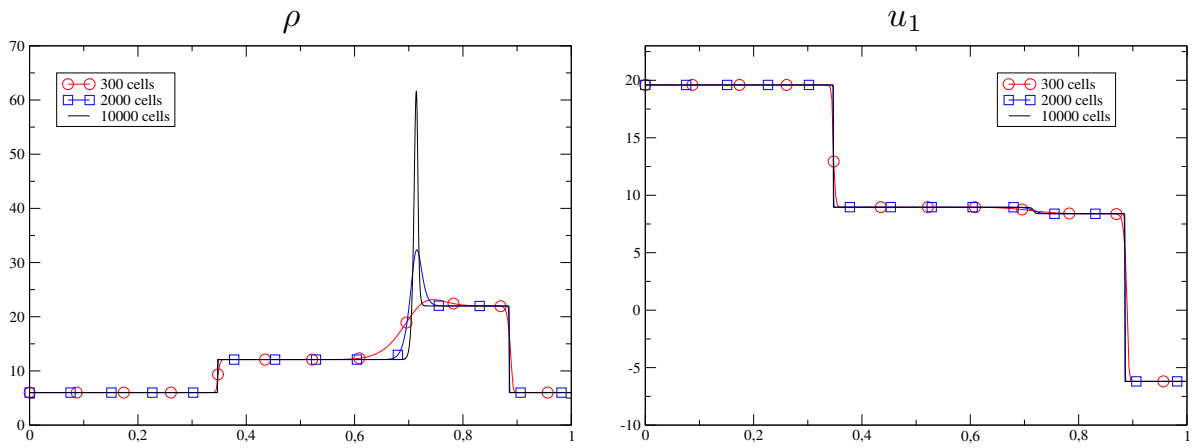

$T_{e}$
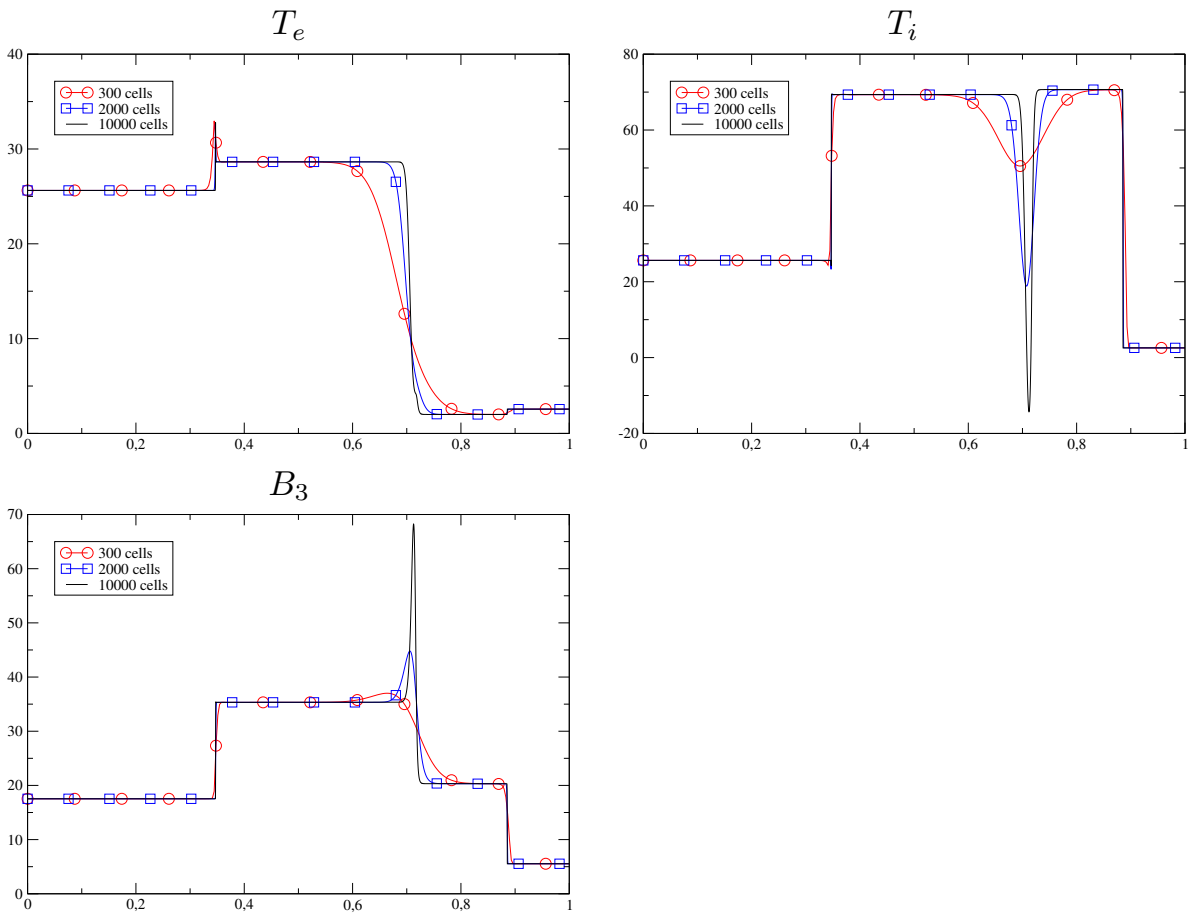

Figure 10: Nonconservative HLL method applied to Test 4 with $x_{0}=0.4$. Numerical solutions are compared at computational time $t=0.035$. 
Nonconservative HLL density
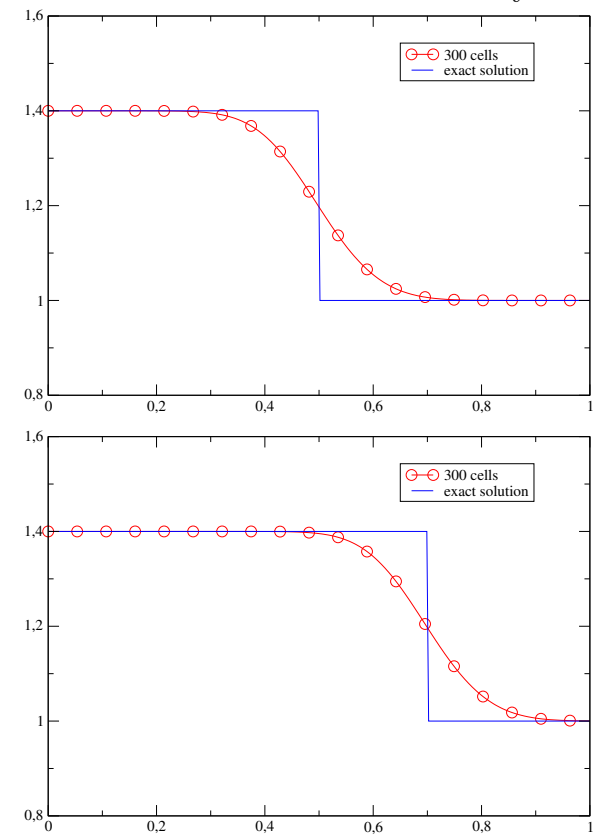

Suliciu density
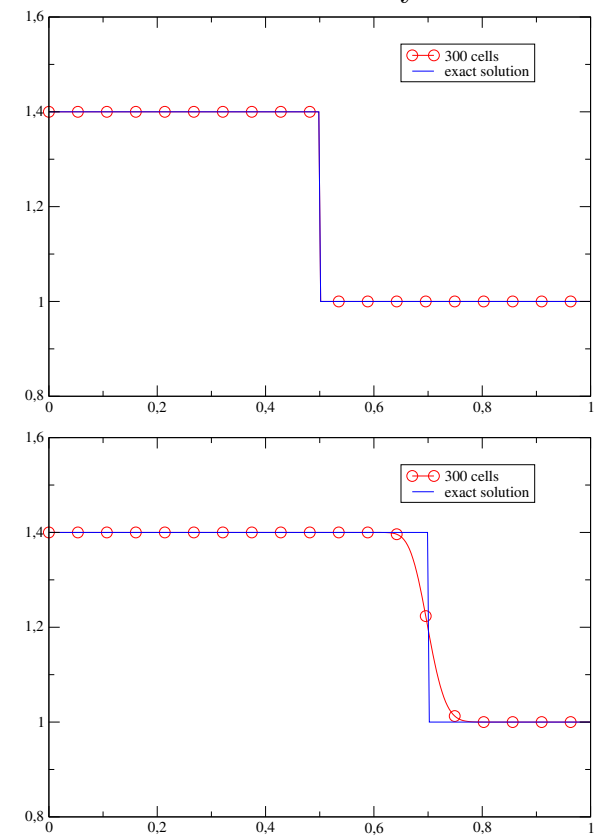

Figure 11: Noncervative HLL method (left) and Suliciu method (right) applied to Test 6 with $x_{0}=0.5$, and Test 7 with $x_{0}=0.3$. Numerical and exact solutions are compared at time $t=2.0$ for both Test 6 and Test 7 . 


\section{Acknowledgement}

The autors want to thank Denise Aregba for fruitful discussions that gave rise to this work. This work was partially granted by the Conseil Régional de la région Nouvelle-Aquitaine.

\section{References}

[1] R. Abgrall and S. Karni, A comment on the computation of nonconservative products. J. Comput. Phys 229 (2010), 2759-2763.

[2] D.Aregba-Driollet, J.Breil, S.Brull, B.Dubroca, E.Estibals Modelling and numerical approximation for the nonconservative bitemperature Euler model, ESSAIM: M2AN 52 (2018), 1353-1383.

[3] F. Bouchut, S. Boyaval, A new model for shallow viscoelastic fluids, Math. Models and Meth. in Appl. Sci. 23 (2013), 1479-1526.

[4] C. Berthon, P. Charrier, B. Dubroca, An HLLC scheme to solve the M1 model of radiative transfer in two space dimensions, J. Sci. Comput. 31 (2007), 347-389.

[5] C. Berthon, F. Coquel, and P. LeFloch, Why many theories of shock waves are necessary: Kinetic relations for nonconservative systems, Proc. R. Soc. Edinburgh 142 (2012), 1-37.

[6] D.S. Balsara, M. Dumbser, and R. Abgrall, Multidimensional HLLC Riemann solver for unstructured meshes with application to Euler and MHD flows, J. Comput. Phys 261 (2014), 172-208.

[7] C. Berthon, B. Dubroca, and A. Sangam. A local entropy minimum principle for deriving entropy preserving schemes. SIAM J. Numer. Anal 50 (2012), 468-491.

[8] C. Berthon, B. Dubroca, and A. Sangam. An entropy preserving relaxation scheme for ten-moments equations with source terms. Commun. Math. Sci. 13 (2015), 2119-2154.

[9] F. Bouchut, Nonlinear stability of finite volume methods for hyperbolic conservation laws, and well-balanced schemes for sources, Birkhäuser, 2004.

[10] F. Bouchut, Entropy satisfying flux vector splitting and kinetic BGK models, Numer. Math. 94 (2003), 623-672.

[11] F. Bouchut, C. Klingenberg, K. Waagan, A multiwave approximate Riemann solver for ideal MHD based on relaxation I - Theoretical framework, Numer. Math. 108 (2007), 7-42.

[12] F. Bouchut, C. Klingenberg, K. Waagan, A multiwave approximate Riemann solver for ideal MHD based on relaxation II - numerical implementation with 3 and 5 waves, Numer. Math. 115 (2010), 647-679.

[13] F. Bouchut, X. Lhébrard, A 5-wave relaxation solver for the shallow water MHD system, J. Scientific Comput., 68 (2016), 92-115. 
[14] C. Chalons, F. Coquel, C. Marmignon, Well-balanced time implicit formulation of relaxation schemes for the Euler equations, SIAM J. Sci. Comput. 30 (2007), 394-415.

[15] C. Chalons, F. Coquel, Modified Suliciu relaxation system and exact resolution of isolated shock waves, Math. Models Methods Appl. Sci. 24 (2014), 937-971.

[16] F. Coquel, E. Godlewski, N. Seguin, Relaxation of fluid systems, Math. Models Methods Appl. Sci. 22 (2012), 1250014.

[17] F. Coquel, and C. Marmignon, Numerical methods for weakly ionized gas, Astrophys. Space Sci. 260 (1998), 15-27.

[18] G. Dal Maso, P. G Lefloch, and F. Murat, Definition and weak stability of nonconservative products, J. Math. Pures Appl. 74 (1995), 483-548.

[19] J. M Greene, Improved Bhatnagar-Gross-Krook model of electron-ion collisions, Phys. Fluids 16 (1973), 2022-2023.

[20] F.G. Fuchs, A.D. McMurry, S. Mishra, N.H. Risebro, and K. Waagan, $A p-$ proximate Riemann Solvers and Robust High-Order Finite Volume Schemes for Multi-Dimensional Ideal MHD Equations, Comm. Comp. Phys. 9 (2011), 324-362.

[21] A. Harten, P.D. Lax, B. Van Leer, On upstream differencing and Godunovtype schemes for hyperbolic conservation laws, SIAM Review 25 (1983), $35-61$.

[22] C. Pares, Numerical methods for nonconservative hyperbolic systems: a theoretical framework. SIAM J. Numer. Anal. 44 (2006), 300-321.

[23] M. Pelanti and K. Shyue, A mixture-energy-consistent six-equation two phase numerical model for fluids with interfaces, cavitation and evaporation waves, J. Comput. Phys. 259 (2014), 331-357.

[24] A. Sangam, An HLLC scheme for ten-moments approximation coupled with magnetic field, Int. J. Comput. Sci. Math. 2 (2008), 73-109.

[25] R. Saurel, F. Petitpas and R. Abgrall, Modelling phase transition in metastable liquids: application to cavitating and flashing flows, J. Fluid Mech. Vol. 607 (2006), 313-350.

[26] R. Saurel, F. Petitpas, R.A. Berry, Simple and efficient relaxation methods for interfaces separating compressible fluids, cavitating flows and shocks in multiphase mixtures, J. Comput. Phys. 228 (2009), 1678-1712.

[27] I. Suliciu, Some stability-instability problems in phase transitions modelled by piecewise linear elastic or viscoelastic constitutive equations, Internat. J. Engrg. Sci. 30 (1992), 483-494.

[28] E. Tadmor, A minimum entropy principle in the gas dynamics equations, Appl. Numer. Math. 2 (1986), 211-219. 
[29] E. Toro, Riemann Solvers and Numerical Methods for Fluid Dynamics: A Practical Introduction

[30] K. Waagan, C. Federrath, C. Klingenberg, A robust numerical scheme for highly compressible magnetohydrodynamics: Nonlinear stability, implementation and tests, J. Comput. Phys. 2030 (2011), 3331-3351. 